\title{
CODIMENSION 1 ORBITS AND SEMI-INVARIANTS FOR THE REPRESENTATIONS OF AN EQUIORIENTED GRAPH OF TYPE $D_{n}$ BY
}

\author{
S. ABEASIS ${ }^{1}$
}

\begin{abstract}
We consider the Dynkin diagram $D_{n}$ equioriented and the variety $\operatorname{Hom}\left(V_{1}, V_{3}\right) \times \prod_{i=2}^{n} \operatorname{Hom}\left(V_{i}, V_{i+1}\right), V_{j}$ a vector space over $K$, on which the group $G=\prod_{i=1}^{n} \mathrm{GL}\left(V_{i}\right)$ acts. We determine the maximal orbit and the codim. 1 orbits of this action, giving their decomposition in terms of the irreducible representations of $D_{n}$. We also deduce a set of algebraically independent semi-invariant polynomials which generate the ring of semi-invariants.
\end{abstract}

0. Introduction. We develop part of a program devoted to study the geometry and the orbit structure for the varieties $L_{d}$ of representations of an oriented graph of finite type ( $d$ a fixed dimension) on which a group $G_{d}$ (a product of linear groups) acts naturally.

The case of a Dynkin diagram $\mathscr{A}_{n}$ has been treated in [1-5]. [1, 2] describe the structure of the codim. 1 orbits and determine the corresponding ring of semi-invariants and $[3,4]$ give a combinatorial description of the closures of the orbits in $L_{d}$ for any orientation of $\mathscr{A}_{n}$. In [5] it is proved, when $\mathscr{A}_{n}$ is equioriented, that the closures of the orbits are algebraic varieties which are normal, Cohen-Macaulay with rational singularities.

We begin the corresponding study for the Dynkin diagram $D_{n}$. In particular we are interested in the description of the codim. 1 orbits. Once we have a "combinatorial" method to produce the set $\mathcal{O}_{1}$ of codim. 1 orbits, it is not difficult to find, using the same method of [1 and 2], the reduced equations for the Zarisky closure $\bar{O}$ of any $O \in O_{1}$; in particular we can deduce a set of algebraically independent semi-invariant polynomials which generate the ring of semi-invariants (cf. §6).

We briefly explain the procedure allowing us to describe the set of codim. 1 orbits $\mathcal{O}_{1}$ when $D_{n}$ is equioriented. Consider the graph $D_{n}$ :

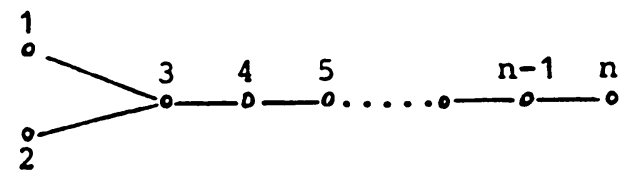

Received by the editors November 5, 1982.

1980 Mathematics Subject Classification. Primary 14L30; Secondary 14D25.

Key words and phrases. Dynkin diagrams, representations, orbits, semi-invariants.

${ }^{1}$ The author belongs to the group GNSAGA of the C.N.R. Part of this paper was worked out while the author was visiting the Mathematics Department of Massachusetts Institute of Technology. 
where $\Gamma_{0}=\{1,2, \ldots, n\}$ is the set of its vertices, and $\Gamma_{1}$ the set of its edges, and let $\Omega$ be an arbitrary orientation for its edges. For any dimension

$$
d=\left(\begin{array}{l}
d_{1} \\
d_{2}
\end{array} ; d_{3}, \ldots, d_{n}\right)
$$

$d_{i}$ nonnegative integers, let $V_{i}$ be a vector space of dimension $d_{i}$ over a given field $K$ and consider the variety of the representations of the oriented graph $\left(D_{n}, \Omega\right)$ :

$$
L_{d}\left(D_{n}, \Omega\right):=\prod_{l \in \Gamma_{1}} \operatorname{Hom}_{K}\left(V_{i(l)}, V_{f(l)}\right)
$$

where $i(l), f(l) \in \Gamma_{0}$ are, respectively, the initial and the final vertex of $l$ with respect to the orientation $\Omega$. The group $G_{d}:=\prod_{i=1}^{n} \operatorname{GL}\left(V_{i}\right)$ acts naturally on $L_{d}\left(D_{n}, \Omega\right)$ and the orbits $O_{B}$ of this action correspond to the isomorphism classes [ $B$ ] of the representations $B \in L_{d}\left(D_{n}, \Omega\right)$. Recall now that the indecomposable representations of $\left(D_{n}, \Omega\right)$ are in 1-1 correspondence with the set $\Delta^{+}$of the positive roots of the Dynkin diagram $D_{n}$, independently from the orientation (cf. [6, 8], and $\S 1$ for an explicit and graphic description). Let us denote them by $E_{\alpha}, \alpha \in \Delta^{+}\left(\Delta^{+}\right.$is finite as $D_{n}$ is of finite type). Then up to isomorphism we have $B=\oplus_{\alpha \in \Delta^{+}} e_{\alpha}^{B} E_{\alpha}$, where $e_{\alpha}^{B}$ is the multiplicity of $E_{\alpha}$ in $B \in L_{d}\left(D_{n}, \Omega\right)$. It follows that the sets $e^{B}=\left\{e_{\alpha}^{B}, \alpha \in \Delta^{+}\right\}$parametrize the orbits $O_{B} \subset L_{d}\left(D_{n}, \Omega\right)$ provided they satisfy the equalities corresponding to the fact that the dimension $d$ is fixed; but their independence from the orientation makes them unsuitable for studying problems which do depend on the orientation, e.g. the structure of the orbits, in particular the description of the maximal orbit and the codim. 1 orbits.

What we do here is choose a special orientation for $D_{n}$, the equiorientation, namely:

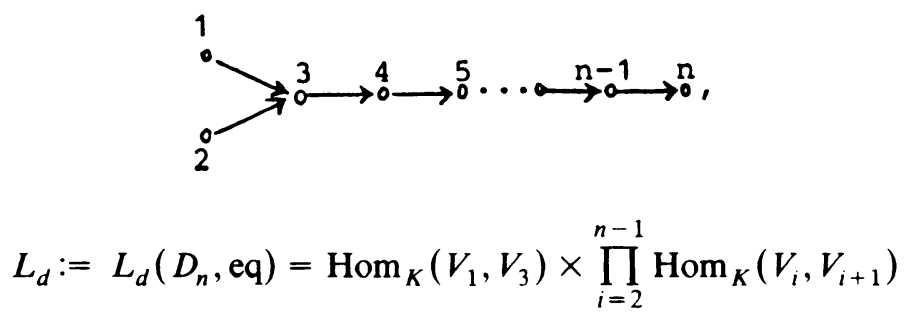

and we reparametrize the orbits with sets of " rank parameters" $N^{B}=\left\{N_{\alpha}^{B}, \alpha \in \Delta^{+}\right\}$, where $N_{\alpha}^{B}$ is the rank of a suitable map $\varphi_{\alpha}^{B}$ constructed from the representation $B \equiv\left(B_{1}, B_{2}, \ldots, B_{n-1}\right)$ :

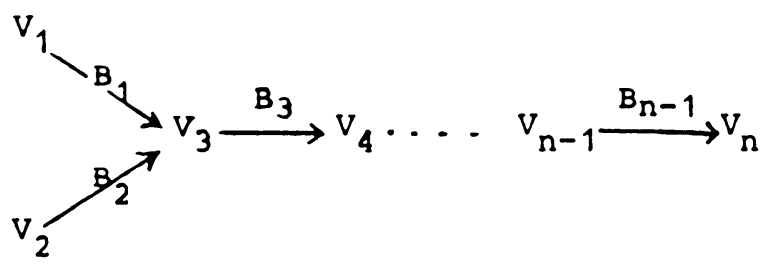


The choice of the maps $\varphi_{\alpha}^{B}$ is related to the geometry of the subspaces given by the images and the kernels of the linear maps $B_{i}$ and their compositions (cf. §1), therefore they need to be changed if we change the orientation of $D_{n}$.

The advantage of this parametrization is the fact that if $B, C \in\left(D_{n}\right.$, eq $)$ and $O_{C} \subset \bar{O}_{B}\left(\bar{O}_{B}\right.$ the Zariski closure of $\left.O_{B}\right)$, then $N_{\alpha}^{C} \leqslant N_{\alpha}^{B}$ for every $\alpha \in \Delta^{+}$, since in a degeneration ranks cannot increase. It follows that the unique maximal orbit $O_{\max } \subset$ $L_{d}\left(D_{n}\right.$, eq) must correspond to a set $N^{A}=\left\{N_{\alpha}^{A}, \alpha \in \Delta^{+}\right\}, A \in O_{\max }$, where the $N_{\alpha}^{A}$ 's are "maximum possible". The construction of $O_{\max }$ is suggested now by this property. In $\$ 3$ we describe explicitly, using a "combinatorial" argument, the indecomposables appearing as factors of $A \in O_{\max }$; equivalently, for any dimension $d($ see $(*))$ we give a combinatorial description of the "canonical decomposition" of the dimension vector $d$ with respect to the chosen orientation of $D_{n}$ (for this notion cf. $[11,12])$.

The classification of the codim. 1 orbits in $L_{d}\left(D_{n}\right.$, eq $)$ is explicitly given in Theorem 5.1. More precisely, for any $d$ as in (*) we define a subset $I_{d}$ of pairs of indices $(i, j), i \gtrless j$ (cf. §4), then we find a bijection $\psi: I_{d} \rightarrow \mathcal{O}_{1}, \mathcal{O}_{1}$ the set of codim. 1 orbits in $L_{d}\left(D_{n}\right.$, eq),$\psi(i, j)=O_{B_{i}}$, where $B_{i j}$ is explicitly constructed in terms of its indecomposable factors. As a consequence of properties (a) and (b) which we establish for $\psi$ in Theorem 5.1, we deduce, for any $O_{B_{i}} \in \mathcal{O}_{1}$, the equation $D_{i j}=0$ for the corresponding algebraic variety $\bar{O}_{B_{1}}$ (cf. §6). A result due to Sato and Kimura (cf. [13]) allows us to describe the ring of semi-invariants $\mathscr{R}_{d}\left(D_{n}\right.$, eq), namely if $K$ is an infinite field, then the $D_{i j}$ 's, $(i, j) \in I_{d}$, are a set of algebraically independent semi-invariant polynomials which generate the ring of semi-invariants $\mathscr{R}_{d}\left(D_{n}\right.$, eq) (cf. $\left.\S 6\right)$.

At this point the proposed problem of classifying the codim. 1 orbits and describing the ring of semi-invariants for any dimension $d$ is solved for $\left(D_{n}\right.$, eq). If we change the orientation, i.e. we consider $\left(D_{n}, \Omega\right)$ for any $\Omega$, and want to solve the same type of problems, we must define a suitable set of maps $\varphi_{\alpha}^{B}, \alpha \in \Delta$, for $B \in L_{d}\left(D_{n}, \Omega\right)$ in such a way that the corresponding ranks parametrize the orbits. Then we can go through the whole procedure developing combinatorial arguments, as in $\S \S 3-5$, suitable for the orientation $\Omega$. The argument will not be trivial, since it is not trivial for the Dynkin diagram $\mathscr{A}_{n}$ (cf. $[1,2]$ ), and in any case will use complicated notation related to the sequence of sources and sinks describing the orientation $\Omega$. We do not do it.

On the other hand, the canonical decomposition of $d$ w.r.t. $\Omega$ does not strictly need knowledge of the "rank parameters" once it is known for a special orientation. In fact any orientation $\Omega$ can be obtained from a given one by performing a suitable number of simple reflections in admissible vertices (cf. [6]), therefore the reflection functors suggest a possible construction of $O_{\max } \subset L_{d}\left(D_{n}, \Omega\right)$. In any case the formal proof that we have produced the maximal orbit is deducible from the computation of the dimension of its stabilizer (once we have computed $\operatorname{dim} \operatorname{Hom}_{K}\left(E_{\alpha}, E_{\beta}\right)$ for any $\alpha, \beta \in \Delta^{+}$and for any orientation $\Omega$ ).

We can also control what happens to the ring of semi-invariants under a change of orientation by using the isomorphisms (2.19) given in [11] (cf. the discussion given in $[2,87])$. 
1. Rank parameters for the representations of $\left(D_{n}\right.$, eq $)$. Consider an equioriented Dynkin diagram $D_{n}$, i.e.

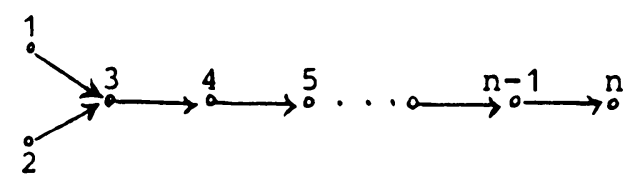

where $\Gamma_{0}=\{1,2,3, \ldots, n\}$ is the set of its vertices. The orientation induces a partial ordering $\leqslant$ in $\Gamma_{0}$ which is the usual ordering in the set $N$ of natural numbers except for the integers 1 and 2, which are incomparable. We will systematically use this ordering, therefore, for example, the interval $[1, q] \subset N$ does not contain the integer 2.

The subdiagram $\mathscr{A}_{n-1}^{(1)}$ relative to the vertices $\{2,3, \ldots, n\}$ is an equioriented Dynkin diagram of type $\mathscr{A}_{n-1}$. Similarly we have $\mathscr{A}_{n-1}^{(2)}$ erasing the vertex 1 .

Recall now that the set of the indecomposable representations of $D_{n}$ is in 1-1 correspondence with the set $\Delta^{+}$of the positive roots of $D_{n}$, independently of the orientation (cf. $[6,8]$ ). We denote by $E_{p q}, p \leqslant q$ (in the ordering specified above), $p, q=1,2, \ldots, n$, the indecomposable representations of $D_{n}$ which are also indecomposable representations either of $\mathscr{A}_{n-1}^{(1)}$ or $\mathscr{A}_{n-1}^{(2)}$, and we denote the remaining ones by $E_{p q}, 3 \leqslant q<p \leqslant n+1$, i.e. the indecomposable representations of $D_{n}$ which are representations neither of $\mathscr{A}_{n-1}^{(1)}$ nor $\mathscr{A}_{n-1}^{(2)}$. Table 1 lists explicitly the dimensions of the $E_{p q}$ 's and gives a "graphic" description of them which will be useful in the future. We have to read the graphic description of the indecomposable $E_{p q}$ as representations for the given orientation. The dots $j$ or $j^{\prime}, j^{\prime \prime}$ always represent a given basis for the corresponding vector space. If $E_{p q}$ is such that $p \leqslant q$, the representation (a canonical form of it) is described by sending the vector $j$ to $j+1, p \leqslant j<q$, and $q$ to zero.

If $E_{p q}$ is such that $p>q$, the representation is described by:

$$
\begin{aligned}
& 1 \rightarrow 3^{\prime}, 2 \rightarrow 3^{\prime \prime} ; j^{\prime} \rightarrow(j+1)^{\prime}, j^{\prime \prime} \rightarrow(j+1)^{\prime \prime} \quad \text { if } 3 \leqslant j<q-1 ; \\
& (q-1)^{\prime} \rightarrow \frac{1}{2} q,(q-1)^{\prime \prime} \rightarrow \frac{1}{2} q ; j \rightarrow j+1 \text { if } q \leqslant j<p-1 ; \\
& p-1 \rightarrow 0 .
\end{aligned}
$$

Note that the graphic description of $E_{p q}$ gives rise to an indecomposable ending at $q$ if $p \leqslant q$, and $p-1$ if $p>q$. From this point of view we will also say that the indecomposable $E_{p q}(p \gtreqless q)$ contains (or passes through) the vertex $j$ if its graphic description contains the vertex $j$ (or $j^{\prime}, j^{\prime \prime}$ ).

Let $A$ be a representation of $\left(D_{n}\right.$, eq). Then $A$ uniquely decomposes, up to isomorphism, into indecomposable factors

$$
[A]=\bigoplus_{p, q} e_{p q}^{A} E_{p q},
$$

where $e_{p q}^{A}$ denotes the multiplicity of $E_{p q}, p \leqslant q \leqslant n, p=1,2, \ldots, n$ or $3 \leqslant q<p \leqslant$ $n+1$. 
TABLE 1. List of the indecomposables of $D_{n}$

\begin{tabular}{|c|c|c|}
\hline$E_{p q}$ & dimension & graphic description \\
\hline $3 \leqslant p \leqslant q \leqslant n$ & 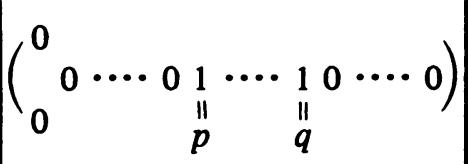 & $\varlimsup_{p} q$ \\
\hline $1=p \leqslant q \leqslant n$ & 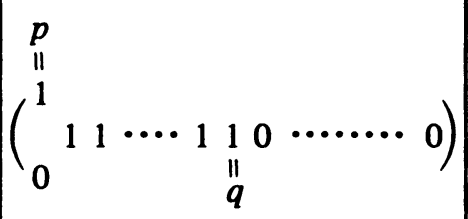 & $\sqrt[1]{3}$ \\
\hline $2=p \leqslant q \leqslant n$ & 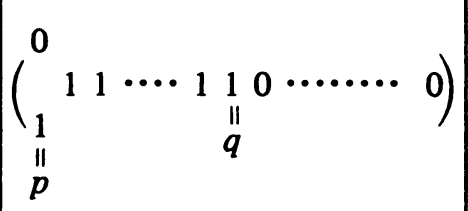 & $\sqrt{3}$ \\
\hline$n+1 \geqslant p>q>3$ & $\left(\begin{array}{l}1 \\
2 \\
1\end{array}\right.$ & $\overbrace{2}^{1} \overbrace{3^{\prime \prime} j^{\prime \prime}(q-1)^{\prime \prime}}^{j^{\prime}(p-1)^{\prime}}$ \\
\hline $\begin{array}{c}n+1 \geqslant p>q=3 \\
p \neq 4\end{array}$ & 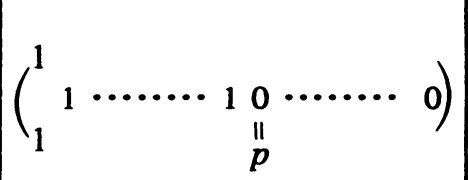 & $\sum_{2}^{1} \bar{j} p-1$ \\
\hline$p=4, q=3$ & 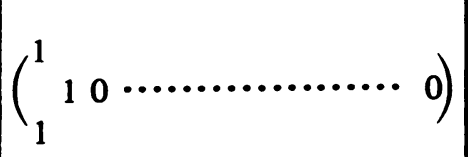 & \\
\hline
\end{tabular}


Conversely a set of nonnegative integers $\left\{\boldsymbol{e}_{p q}\right\}$ determines a unique isomorphism class $[A]$ of representations of $\left(D_{n}\right.$, eq). Therefore the isomorphism classes of representations of our oriented graph are parametrized by the sets of nonnegative integers $\left\{e_{p q}\right\}, p \leqslant q \leqslant n, p=1,2, \ldots, n$, or $3 \leqslant q<p \leqslant n+1$.

We now fix a dimension $d$ as in $(*)$ for the representations of $\left(D_{n}\right.$, eq) and consider the vector space

$$
L_{d}:=L_{d}\left(D_{n}, \text { eq }\right)=\operatorname{Hom}_{K}\left(V_{1}, V_{3}\right) \times \prod_{i=2}^{n-1} \operatorname{Hom}_{K}\left(V_{i}, V_{i+1}\right),
$$

i.e. the variety of all representations of dimension $d$ on which the group $G_{d}=$ $\prod_{i=1}^{n} \mathrm{GL}\left(V_{i}\right)$ acts naturally. The orbit $G_{d} \cdot A=O_{A}$ of an element $A \in L_{d}$ coincides with the isomorphism class $[A]$ of $A$, and the given action of $G_{d}$ on $L_{d}$ has a finite number of orbits and a unique maximal orbit (as it follows from the fact that the number of indecomposable representations is finite).

We now want to describe a different parametrization of the orbits in $L_{d}\left(D_{n}\right.$, eq).

Consider first the situation when $n=4$ :

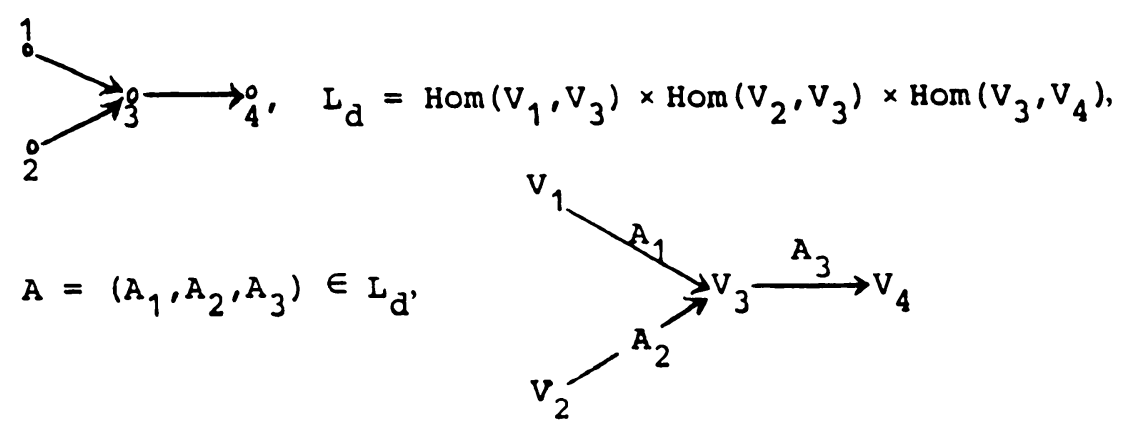

Starting from the three linear maps $A_{i}$ we can construct the following five linear maps:

$$
\begin{array}{ll}
V_{1} \rightarrow V_{4}, & v_{1} \nrightarrow A_{3} A_{1} v_{1}, \\
V_{2} \rightarrow V_{4}, & v_{2} \nrightarrow A_{3} A_{2} v_{2}, \\
V_{1} \oplus V_{2} \rightarrow V_{3}, & \left(v_{1}, v_{2}\right) \nrightarrow\left(A_{1} v_{1}+A_{2} v_{2}\right), \\
V_{1} \oplus V_{2} \rightarrow V_{4}, & \left(v_{1}, v_{2}\right) \nrightarrow\left(A_{3} A_{1} v_{1}, A_{3} A_{2} v_{2}\right), \\
V_{1} \oplus V_{2} \oplus V_{3} \rightarrow\left(V_{3} \oplus V_{3} \oplus V_{3}\right) / \Delta \oplus V_{4},
\end{array}
$$

where $\Delta$ is the diagonal in $V_{3} \oplus V_{3} \oplus V_{3}$ and the linear map is defined by

$$
\left(v_{1}, v_{2}, v_{3}\right) \leadsto\left(\left(\overline{A_{1} v_{1}, A_{2} v_{2}, v_{3}}\right), A_{3} v_{3}\right),
$$

where $\left(\overline{A_{1} v_{1}, A_{2} v_{2}, v_{3}}\right)$ is the class of $\left(A_{1} v_{1}, A_{2} v_{2}, v_{3}\right)$ modulo the diagonal.

Note that if we include the identity map $V_{i} \rightarrow V_{i}, i=1, \ldots, 4$, then we have 12 linear maps, as many as the number of indecomposable representations of $D_{4}$.

We are interested in computing the rank of these maps (the reason will be clear from the discussion of the general case; cf. Proposition 1.4). In particular, we want to compute them in terms of the dimensions of the subspaces $\operatorname{Im} A_{1}, \operatorname{Im} A_{2}, \operatorname{ker} A_{3}$ $\left(\subset V_{3}\right), \operatorname{Im} A_{3} A_{1}, \operatorname{Im} A_{3} A_{2}\left(\subset V_{4}\right)$, and their intersections. 
We have

$$
\mathrm{rk}\left(V_{1} \oplus V_{2} \rightarrow V_{3}\right)=\mathrm{rk} A_{1}+\mathrm{rk} A_{2}-\operatorname{dim}\left(\operatorname{Im} A_{1} \cap \operatorname{Im} A_{2}\right)
$$

and similarly for $V_{1} \oplus V_{2} \rightarrow V_{4}$. For the map

$$
\theta: V_{1} \oplus V_{2} \oplus V_{3} \rightarrow\left(V_{3} \oplus V_{3} \oplus V_{3}\right) / \Delta \oplus V_{4}
$$

we have

$$
\begin{aligned}
\text { rk } \theta & =\operatorname{dim} V_{1}+\operatorname{dim} V_{2}+\operatorname{dim} V_{3}-\operatorname{dim} \operatorname{ker} \theta, \\
\operatorname{ker} \theta & =\left\{\left(v_{1}, v_{2}, v_{3}\right): A_{1} v_{1}=A_{2} v_{2}=v_{3}, A_{3} v_{3}=0\right\}
\end{aligned}
$$

Let

$$
\mathscr{C}=\left\{\left(v_{1}, v_{2}\right) \in V_{1} \oplus V_{2}: A_{1} v_{1}=A_{2} v_{2} \in \operatorname{ker} A_{3}\right\} ;
$$

then $\mathscr{C}$ is canonically isomorphic to $\operatorname{ker} \theta$, and we also have the surjective morphism

$$
\begin{aligned}
\tau: \mathscr{C} \rightarrow W & =\operatorname{Im} A_{1} \cap \operatorname{Im} A_{2} \cap \operatorname{ker} A_{3}, \\
\tau\left(v_{1}, v_{2}\right) & =A_{1} v_{1} \quad\left(=A_{2} v_{2}\right) .
\end{aligned}
$$

It follows that

$$
\begin{aligned}
\operatorname{dim} \operatorname{ker} \theta= & \operatorname{dim} \mathscr{C}=\operatorname{dim}\left(\operatorname{Im} A_{1} \cap \operatorname{Im} A_{2} \cap \operatorname{ker} A_{3}\right)+\operatorname{ker} \tau \\
= & \operatorname{dim}\left(\operatorname{Im} A_{1} \cap \operatorname{Im} A_{2} \cap \operatorname{ker} A_{3}\right)+\left(\operatorname{dim} V_{1}-\operatorname{rk} A_{1}\right) \\
& +\left(\operatorname{dim} V_{2}-\operatorname{rk} A_{2}\right)
\end{aligned}
$$

and

rk $\theta=\operatorname{dim} V_{3}+$ rk $A_{1}+$ rk $A_{2}-\operatorname{dim}\left(\operatorname{Im} A_{1} \cap \operatorname{Im} A_{2} \cap \operatorname{ker} A_{3}\right)$.

We return to the general case. Let $A=\left(A_{1}, A_{2}, \ldots, A_{n-1}\right) \in L_{d}\left(D_{n}\right.$, eq $)$ :

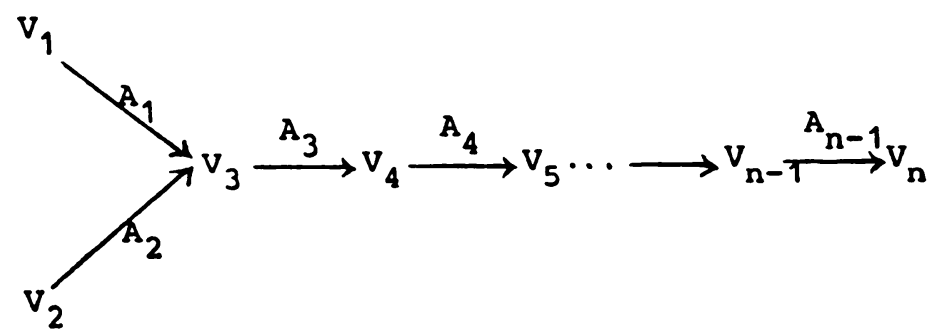

For any pair of indices $w, z \in[3, n], w<z$, we consider the "contracted diagram" $D_{4}$ of vertices $1,2, w, z$,

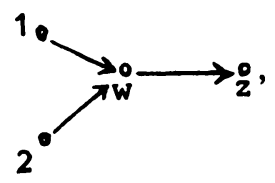

the contracted representation obtained from $A$ via the obvious composition of the $A_{i}$ 's and the linear maps defined above (not all of them are distinct). We deduce the following distinct linear maps associated to $A$ : 
DEFINITIONS AND Notations.

(1)

$$
\begin{aligned}
& \varphi_{h k}^{A}=A_{k-1} \cdots A_{h+1} A_{h}: V_{h} \rightarrow V_{k}, \quad 2 \leqslant h<k \leqslant n, \\
& \varphi_{1 k}^{A}=A_{k-1} \cdots A_{3} A_{1}: V_{1} \rightarrow V_{k}, \quad k \leqslant n .
\end{aligned}
$$

(2)

$$
\begin{aligned}
& \varphi_{n+1, k}^{A}: V_{1} \oplus V_{2} \rightarrow V_{k}, \quad 3 \leqslant k \leqslant n, \\
& \varphi_{n+1, k}^{A}\left(v_{1}, v_{2}\right)=\varphi_{1 k}^{A} v_{1}+\varphi_{2 k}^{A} v_{2} .
\end{aligned}
$$

(3)

$$
\begin{aligned}
& \varphi_{h k}^{A}: V_{1} \oplus V_{2} \oplus V_{k} \rightarrow\left(V_{k} \oplus V_{k} \oplus V_{k}\right) / \Delta \oplus V_{h}, \quad 3 \leqslant k<h \leqslant n, \\
& \varphi_{h k}^{A}\left(v_{1}, v_{2}, v_{k}\right)=\left(\left(\overline{\varphi_{1 k}^{A} v_{1}, \varphi_{2 k}^{A} v_{2}, v_{k}}\right), \varphi_{k h}^{A} v_{k}\right) .
\end{aligned}
$$

We now define $N^{A}=\left\{N_{h k}^{A}\right\}$, the set of "rank parameters" of the representation A.

DEFINITION.

(1')

$$
\begin{aligned}
& N_{h k}^{A}=\text { rk } \varphi_{h k}^{A}, \quad \text { if } h<k, h, k=1, \ldots, n, \\
& N_{h k}^{A}=d_{h}, \quad \text { if } h=k, h, k=1, \ldots, n .
\end{aligned}
$$

$\left(2^{\prime}\right)$

$$
\begin{aligned}
N_{n+1, k}^{A} & =\mathrm{rk} \varphi_{n+1, k}^{A} \\
& =\mathrm{rk} \varphi_{1 k}^{A}+\mathrm{rk} \varphi_{2 k}^{A}-\operatorname{dim}\left(\operatorname{Im} \varphi_{1 k}^{A} \cap \operatorname{Im} \varphi_{2 k}^{A}\right), \quad \text { if } 3 \leqslant k \leqslant n .
\end{aligned}
$$

$$
\begin{aligned}
N_{h k}^{A} & =\mathrm{rk} \varphi_{h k}^{A}-\operatorname{dim} V_{k} \\
& =\operatorname{rk} \varphi_{1 k}^{A}+\mathrm{rk} \varphi_{2 k}^{A}-\operatorname{dim}\left(\operatorname{Im} \varphi_{1 k}^{A} \cap \operatorname{Im} \varphi_{2 k}^{A} \cap \operatorname{ker} \varphi_{k h}^{A}\right) \quad \text { if } 3 \leqslant k<h \leqslant n .
\end{aligned}
$$

The integers $N_{h k}^{A}$ are all nonnegative. Clearly the same set $N^{A}$ is associated to all the representations of the isomorphism class $[A]=O_{A}$; moreover, the number of elements in $N^{A}$ equals the number of nonisomorphic indecomposable representations of $D_{n}$. Note that the number $N_{n+1, k}^{A}$ defined in (2') can be considered a "special case" of $\left(3^{\prime}\right)$ (it is enough to extend $D_{n}$ by adding a vertex $n+1$ and to assume $d_{n+1}=0$ ).

Now consider the decomposition

$$
A=\sum e_{p q}^{A} E_{p q} .
$$

Clearly we can express the rank parameters $N_{h k}^{A}(h \gtreqless k)$ in terms of the multiplicities $e_{p q}^{A}$, as ranks are additive (and the dimension too), i.e. we have a linear system

$$
N_{h k}^{A}=f_{h k}\left(e_{p q}^{A}\right) \text {. }
$$


We want to show that (1.1) is invertible over the integers. For this we introduce the auxiliary set of parameters $m^{A}=\left\{m_{p q}^{A}\right\}$ associated to $[A]=O_{A}$ and define as follows:

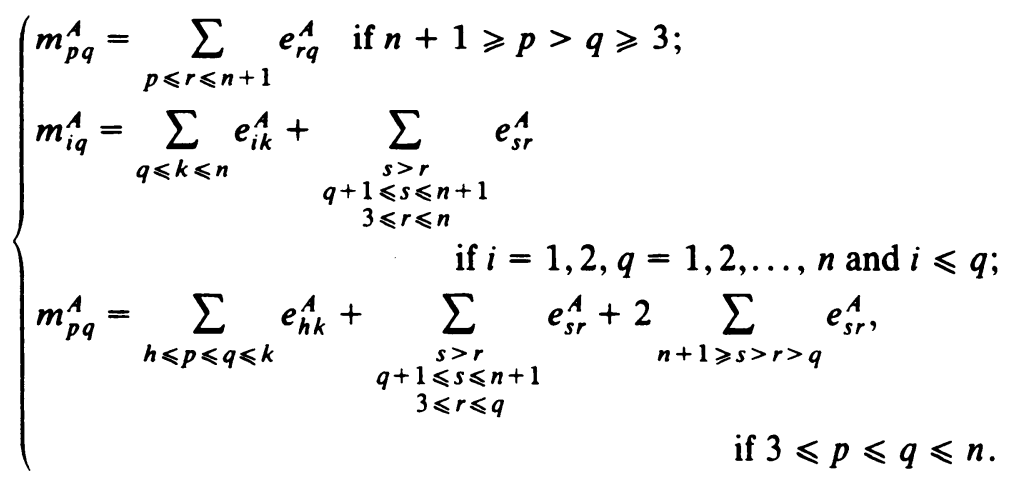

The linear system (1.2) can be inverted over the integers, in fact the equations of (1.2) are recursive. It follows that we have the relations

$$
e_{p q}^{A}=g_{p q}\left(m_{u v}^{A}\right)
$$

which imply that a set $m=\left\{m_{u v}\right\}$ of nonnegative integers determines an orbit in $L_{d}$ if and only if $g_{p q}\left(m_{u v}\right) \geqslant 0$.

On the other side it is immediately seen, using the definition of the rank parameters $N_{p q}^{A}$, that we have

$$
\left\{\begin{array}{l}
N_{p q}^{A}=m_{p q}^{A} \text { if } 1 \leqslant p \leqslant q \leqslant n, \\
N_{p q}^{A}=m_{1 q}^{A}+m_{2 q}^{A}-\sum_{j=3, \ldots, q}\left(m_{q+1, j}^{A}-m_{p+1, j}^{A}\right)
\end{array}\right.
$$

which is again a recursive system of linear equations, i.e. it is invertible. (1.1) is obtained by substituting (1.2) in (1.3) and therefore it is invertible over the integers; i.e. we have

$$
e_{p q}^{A}=h_{p q}\left(N_{u v}^{A}\right)
$$

The consequence is the following

Propositron 1.4. (i) $A$ set of nonnegative integers $N=\left\{N_{u v}, u \lesseqgtr v\right\}$ determines the orbit of a representation of $L_{d}\left(D_{n}\right.$, eq) if and only if $h_{p q}\left(N_{u v}\right) \geqslant 0$.

(ii) The sets $N=\left\{N_{u v}\right\}$ satisfying (i) parametrize the orbits in $L_{d}\left(D_{n}, \Omega\right)$.

Proposition 1.5. Let $A, B \in\left(D_{n}\right.$, eq $), O_{B} \subset \bar{O}_{A}$ and $O_{B} \neq O_{A}$. Then $N_{h k}^{B} \leqslant N_{h k}^{A}$ for all pairs $(h, k)$ and the strict inequality holds for at least a pair.

Proof. In a degeneration the rank of the linear maps $\varphi_{h k}(h \lesseqgtr k)$ cannot increase and the dimension $d$ is constant. Moreover, as a consequence of Proposition 1.4(i), we must have at least a strict inequality. 
Assume we know the decomposition $A=\sum_{p q} e_{p q}^{A} E_{p q}$, i.e. we know the multiplicities $e_{p q}^{A}$. Then we can compute $N_{h k}^{A}$ by computing $N_{h k}^{E_{p q}}$ for every $E_{p q}$ such that $e_{p q}^{A}>0$, since ranks are additive. The computation of $N_{h k}^{E_{p q}}$ is immediate for every $p, q, h, k$ by using the graphic description of $E_{p q}$ and definitions $\left(1^{\prime}\right)-\left(3^{\prime}\right)$.

Before ending we want to justify the choice of the orientation of $D_{n}$ given at the beginning of this section.

Consider the Dynkin diagram $D_{n}$ for $n=4$. We have only two possible orientations,
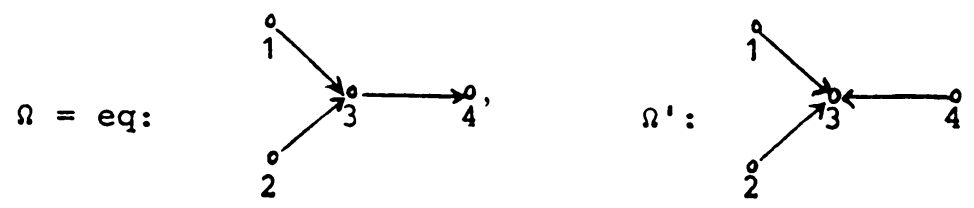

since we can always permute the vertices $(1,2,4)$ or simultaneously reverse all the arrows.

The geometry of the representations of $\left(D_{4}, \Omega^{\prime}\right)$ immediately suggests how to parametrize the orbits of given dimension using rank parameters.

Nevertheless the orientation $\Omega=$ eq priviledged in the sense that we are able to deduce for free the "rank parameters" for the representations of $\left(D_{n}\right.$, eq) from that of $\left(D_{4}, e q\right)$.

In the following sections we will systematically use the oriented graph $\left(D_{n}\right.$, eq).

2. Stabilizer formula. Let $A \in L_{d}\left(D_{n}\right.$, eq) and let $O_{A}$ be the corresponding orbit. Then

$$
\operatorname{dim} O_{A}=\operatorname{dim} G_{d}-\operatorname{dim} \operatorname{St} A
$$

where St $A$ denotes the subgroup of $G_{d}$ which fixes $A$. Moreover, $\operatorname{dim} \operatorname{St} A$ equals $\operatorname{dim} \operatorname{Lie}(\operatorname{St} A)$; therefore

$$
\operatorname{dim} \mathrm{St} A \sum_{p, q, r, s} e_{p q}^{A} e_{r s}^{A} \operatorname{dim} \operatorname{Hom}_{K}\left(E_{p q}, E_{r s}\right) \text {. }
$$

$\operatorname{Hom}_{K}\left(E_{p q}, E_{r s}\right)$ depends only on the integers $p, q, r, s$, as the orientation of $D_{n}$ has been chosen once for all. We collect the possible values of $\lambda=\operatorname{dim} \operatorname{Hom}_{K}\left(E_{p q}, E_{r s}\right)$ in Table 2, which has been constructed case by case using the list of the indecomposables of $\$ 1$. We explain the more interesting cases. For instance consider $\operatorname{Hom}_{K}\left(E_{p q}, E_{r s}\right)$ when $3 \leqslant q<p \leqslant n+1$ and $3 \leqslant s<r \leqslant n+1$. We use the graphic description of the indecomposables and the convention on the dots, explicitly writing only the relevant ones for our discussion. First assume $r \leqslant q$. (See Diagram A.) We have two independent elements $\varphi, \psi \in \operatorname{Hom}_{K}\left(E_{p q}, E_{r s}\right)$, which generate $\operatorname{Hom}_{K}\left(E_{p q}, E_{r s}\right)$ as a vector space, defined as follows:

$$
\begin{aligned}
& \varphi: 1 \mapsto \overline{1}, 3^{\prime} \rightarrow \overline{3}^{\prime}, \ldots,(s-1)^{\prime} \mapsto(\overline{s-1})^{\prime}, s^{\prime} \rightarrow \frac{1}{2} \bar{s}, \ldots,(r-1)^{\prime} \rightarrow \frac{1}{2}(\overline{r-1}) \text {, and } \\
& \text { all other base vectors in } E_{p q} \text { go to zero. } \\
& \psi: 2 \mapsto \overline{2}, 3^{\prime \prime} \mapsto \overline{3}^{\prime \prime}, \ldots,(s-1)^{\prime \prime} \mapsto(\overline{s-1})^{\prime \prime}, s^{\prime \prime} \rightarrow \frac{1}{2} \bar{s}, \ldots,(r-1)^{\prime \prime} \rightarrow \frac{1}{2}(\overline{r-1}) \\
& \quad \text { and all other base vectors in } E_{p q} \text { are sent to zero. (See Diagram B.) }
\end{aligned}
$$




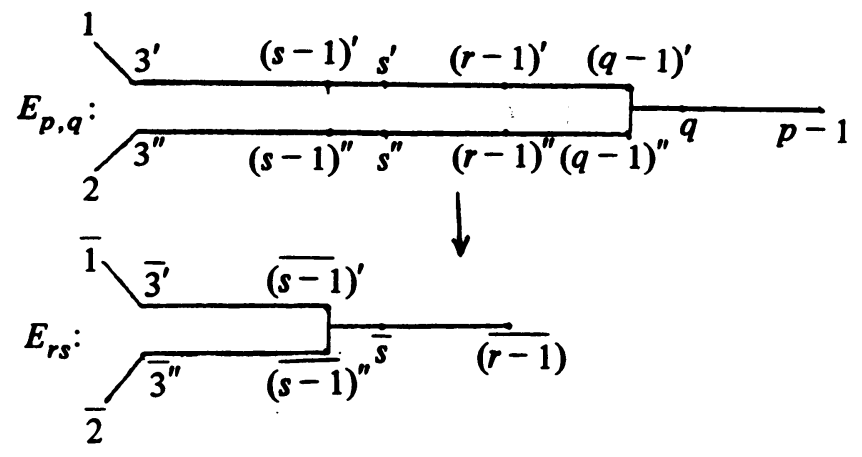

\section{Diagram A}

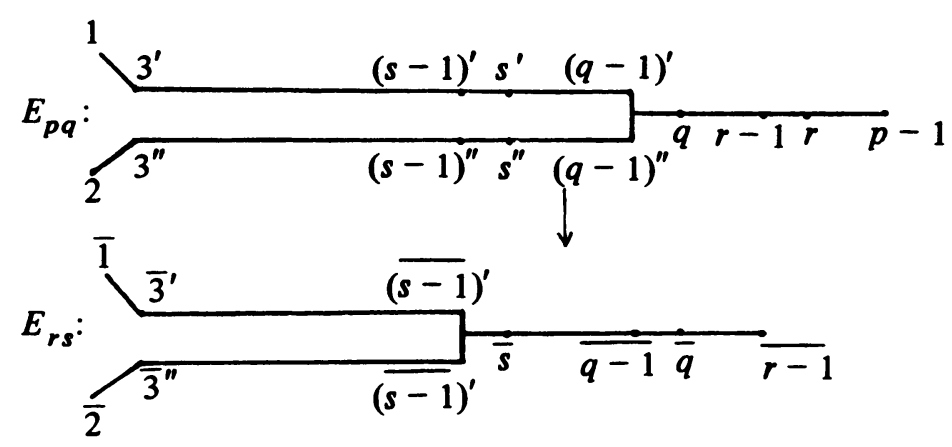

\section{Diagram B}

If $s \leqslant q<r \leqslant p$ we have the following morphism $\psi$ which generates $\operatorname{Hom}_{K}\left(E_{p q}, E_{r s}\right)$ :

$\psi: 1 \mapsto \overline{1}, 2 \mapsto \overline{2}, 3^{\prime} \mapsto \overline{3}^{\prime}, 3^{\prime \prime} \mapsto \overline{3}^{\prime \prime}, \ldots,(s-1)^{\prime} \mapsto(\overline{s-1})^{\prime},(s-1)^{\prime \prime} \mapsto(\overline{s-1})^{\prime \prime}$, $s^{\prime} \rightarrow \frac{1}{2} \bar{s}, s^{\prime \prime} \rightarrow \frac{1}{2} \bar{s}, \ldots,(q-1)^{\prime} \rightarrow \frac{1}{2}(\overline{q-1}), \quad(q-1)^{\prime \prime} \mapsto \frac{1}{2}(\overline{q-1}), q \mapsto$ $\bar{q}, \ldots,(r-1) \mapsto(\overline{r-1})$ and all other base vectors are sent to zero.

In all other possible cases it is easy to see that

$$
\operatorname{Hom}_{K}\left(E_{p q}, E_{r s}\right)=0 \quad(p>q, r>s) .
$$

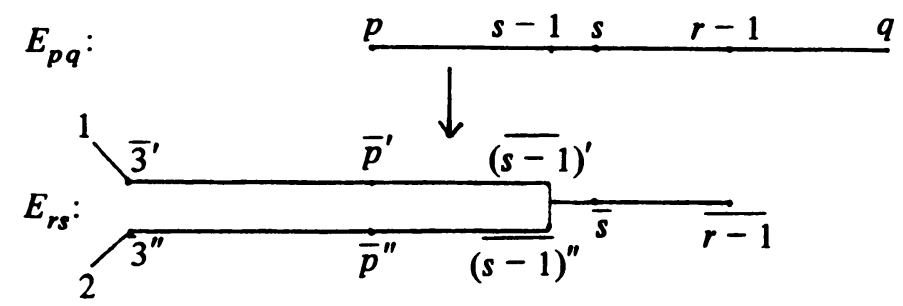


Now consider the case for $E_{p q}$ such that $3 \leqslant p \leqslant q \leqslant n$ and $E_{r s}$ such that $3<s^{p q}<$ $r \leqslant n+1$. (See Diagram C.) If $p<s \leqslant r-1 \leqslant q$ we have two independent elements $\varphi, \psi \in \operatorname{Hom}_{K}\left(E_{p q}, E_{r s}\right)$, which generate it as a vector space, defined as follows:

$\varphi: p \rightarrow \vec{p}^{\prime}, \ldots,(s-1) \rightarrow(\overline{s-1})^{\prime}, s \rightarrow-\frac{1}{2} \bar{s}, \ldots,(r-1) \rightarrow \frac{1}{2}(\overline{r-1})$, and all other base vectors in $E_{p q}$ are sent to zero.

$\psi: p \rightarrow \vec{p}^{\prime}, \ldots,(s-1) \rightarrow(\overline{s-1})^{\prime \prime}, s \rightarrow \frac{1}{2} \bar{s}, \ldots,(r-1) \rightarrow \frac{1}{2}(\overline{r-1})$ and all other base vectors in $E_{p q}$ are sent to zero. (See Diagram D.)

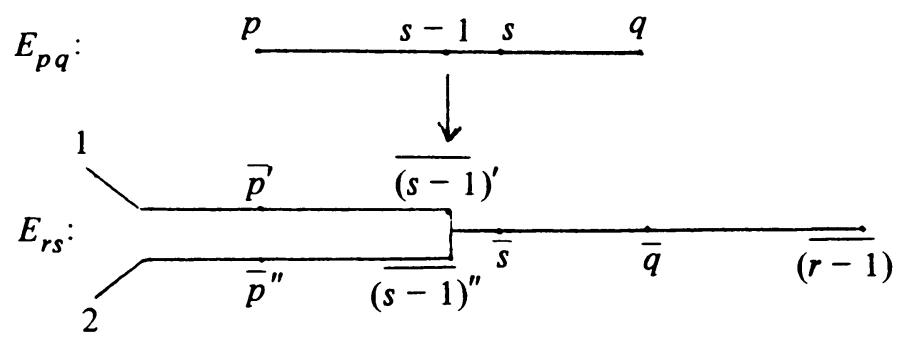

Diagram D

If $p \leqslant s-1 \leqslant q<r-1$, the following $\tilde{\psi}$ generates $\operatorname{Hom}_{K}\left(E_{p q}, E_{r s}\right)$ as a vector space:

$\tilde{\psi}: p \mapsto \vec{p}^{\prime}-\vec{p}^{\prime \prime}, \ldots,(s-1) \mapsto(\overline{s-1})^{\prime}-(\overline{s-1})^{\prime \prime}$ and all the other base vectors of $E_{p q}$ are sent to zero.

In all other possible cases a generator of $\operatorname{Hom}_{K}\left(E_{p q}, E_{r s}\right)$, if any, is forced by the given orientation. In Table 2 we collect all possible values of $\lambda=$ $\operatorname{dim} \operatorname{Hom}_{K}\left(E_{p q}, E_{r s}\right)$. The indecomposables for which $\lambda \neq 0$ are described graphically because it is easier to visualize the relative position of the indices $p, q, r, s$. We do not explicitly consider the case $p=2$ or $r=2$. In fact we clearly have $\operatorname{Hom}_{K}\left(E_{1 q}, E_{2 s}\right)=0$ for all $q, s$, and we can deduce the other possibilities permuting the vertices 1 and 2 in the oriented graph $\left(D_{n}\right.$, eq).

From the table we note that if $E_{p q} \neq E_{r s}$ and $\operatorname{Hom}\left(E_{p q}, E_{r s}\right) \neq 0$, then $\operatorname{Hom}\left(E_{r s}, E_{p q}\right)=0$; moreover,

$$
\lambda=\operatorname{dim} \operatorname{Hom}\left(E_{p q}, E_{r s}\right)=1 \text { if }(p, q)=(r, s) .
$$

Let us define

$$
\left[E_{p q}, E_{r s}\right]=\operatorname{dim} \operatorname{Hom}_{K}\left(E_{p q}, E_{r s}\right)+\operatorname{dim} \operatorname{Hom}_{K}\left(E_{r s}, E_{p q}\right) .
$$

The values of $\left[E_{p q}, E_{r s}\right]$ can be read directly from Table 2 using the obvious symmetrization. In particular, $\left[E_{p q}, E_{p q}\right]=2$.

Let $A$ be a representation of $\left(D_{n}\right.$, eq) and assume $A=B \oplus C$. Then from (2.2) we get

$$
\begin{aligned}
\operatorname{dim} \text { St } A & =\operatorname{dim} \text { St } B+\operatorname{dim} \text { St } C+[B, C] \\
& =\operatorname{dim} \text { St } B+\operatorname{dimSt} C+\sum_{p, q, r, s} e_{p q}^{B} e_{r s}^{C}\left[E_{p q}, E_{r s}\right]
\end{aligned}
$$




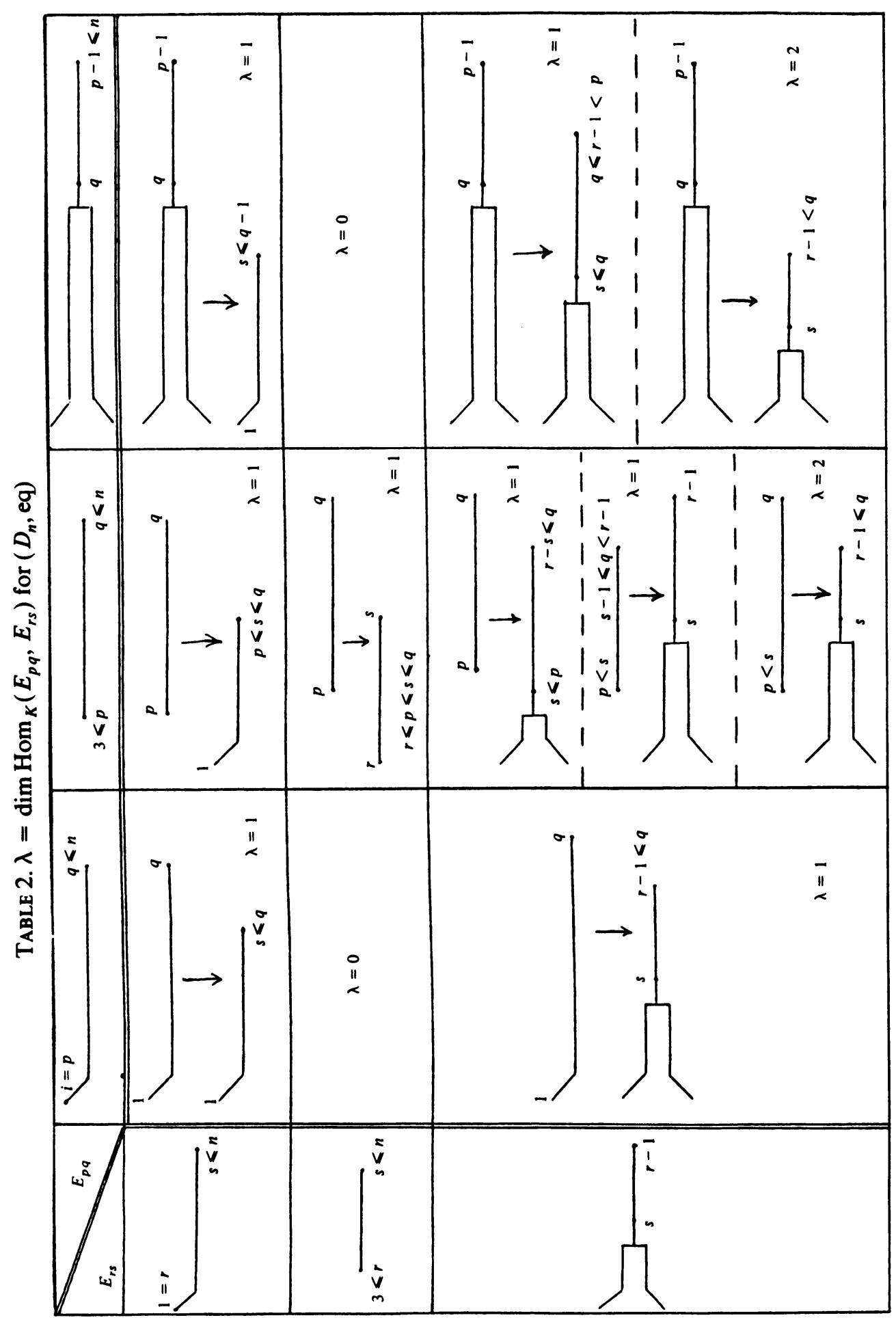


3. The canonical construction. We want to describe a construction $C$ which associates to each $d($ see $(*))$ a special representation $C(d) \in L_{d}\left(D_{n}\right.$, eq), whose main property is given by

Proposition 3.1. Let $A=C(d)$. Then $O_{A}$ is the maximal orbit of $L_{d}\left(D_{n}\right.$, eq).

Construction of $C(d)$. We may always assume $d_{2} \leqslant d_{1}$ (in fact the permutation $(1,2)$ is a graph automorphism of $D_{n}$ which preserves the orientation). The construction is given inductively on the vertex $i$, the first step being, for $i=3$, the construction of the maximal orbit relative to the dimension $\tilde{d}=\left(d_{1}, d_{2}\right)$ for the oriented graph of type $\mathscr{A}_{n}(n=3)$.

$$
\begin{aligned}
& 1 \\
& 0
\end{aligned} \rightarrow \begin{aligned}
& 3 \\
& 0
\end{aligned} \text {. }
$$

The construction was given in [2] for any $n$ and any orientation. We recall the construction since it is very simple.

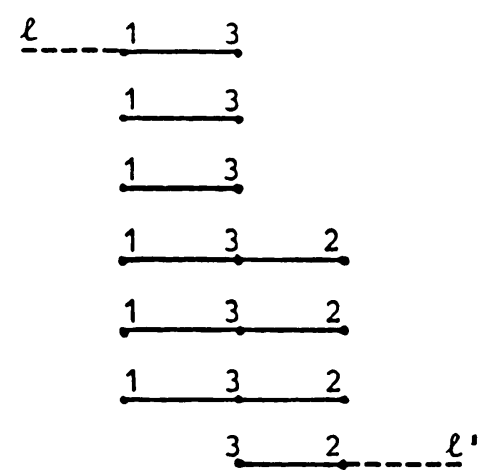

We draw as many dots 1 as $d_{1}$ and, on an "orizontal" line $l$ passing through 1 , as many dots 3 as $d_{3}$ (the first one on the line $l$ ). Then we join dots 1 and 3 (if possible) with an orizontal edge. From the last vertex 3 we draw a line $l^{\prime}$ parallel to $l$, and we draw as many dots 2 as $d_{2}$, (the first one always on $l^{\prime}$ and in the half-plane containing $l$ ), and we proceed as before. The result is a collection of indecomposables for which we use the notations and the graphic description introduced in $§ 1$.

EXAMPLES.

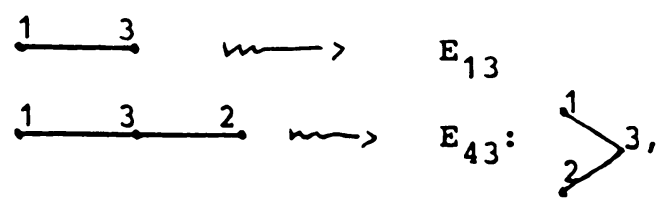

We denote this construction by $C_{3}$. 
Assume we have performed the construction $C_{i}$ up to the vertex $i$ (and for the dimension

$$
\tilde{d}_{i}=\left(\begin{array}{l}
d_{1} \\
d_{2}
\end{array} ; d_{3}, d_{4}, \ldots, d_{i}\right)
$$

$C_{i}$ is the maximal orbit, $i \geqslant 3$, for the subdiagram of vertices $\left.1, \ldots, i\right)$. Then we want to add the vertex $i+1$ and describe the construction $C_{i+1}$. This construction is suggested from the fact that the sets of rank parameters parametrize the orbits (cf. Propositions 1.4, and 1.5), therefore the maximal orbit must correspond to a set with maximum possible rank parameters. It follows that all the indecomposables of $C_{i}$ which do not end at the vertex $i$ are also factors in $C_{i+1}$ (and their multiplicity is unchanged). The construction $C_{i+1}$ will "extend" to $i+1$ some (or all) of the indecomposables of $C_{i}$ which end at the vertex $i$ (the remaining ones being unchanged in $C_{i+1}$ ), according to the following rule.

Assume that in $C_{i}$ we have factors $E_{i+1, t}$ with $i+1>t$ and let us denote by:

$$
\begin{aligned}
& f_{0} \text { the multiplicity of the factor } E_{i+1, i_{0}}\left(\text { in } C_{i}\right) ; \\
& f_{1} \text { the multiplicity of the factor } E_{i+1, i_{1}}\left(\text { in } C_{i}\right) ; \\
& \vdots \\
& f_{r} \text { the multiplicity of the factor } E_{i+1, i_{r}}\left(\text { in } C_{i}\right) \text {, }
\end{aligned}
$$

where $3 \leqslant i_{0}<i_{1}<\cdots<i_{r} \leqslant i$ and in $C_{i}$ there is no factor $E_{i+1, q}$ such that $3 \leqslant q<i_{0}$ or $i_{h}<q<i_{h+1}, h=0, \ldots, r-1$, or $q>i_{r} .\left\{f_{0}, f_{1}, \ldots, f_{r}\right\}$ is a sequence of positive integers (empty if in $C_{i}$ there is no factor $E_{i+1, t}, i+1>t$ ). Moreover consider the following nonnegative integers;

$$
\begin{array}{ll}
k & \text { the number of pairs } E_{1 i} \oplus E_{2 i}\left(\text { in } C_{i}\right), \\
l & \text { the number of the remaining } E_{1 i}\left(\text { in } C_{i}\right), \\
m_{3} & \text { the multiplicity of } E_{3 i}\left(\text { in } C_{i}\right), \\
\vdots & \\
m_{i-1} & \text { the multiplicity of } E_{i-1, i}\left(\text { in } C_{i}\right), \\
m_{i} & \text { the multiplicity of } E_{i i}\left(\text { in } C_{i}\right) .
\end{array}
$$

Let $m=\sum_{p=3}^{i} m_{p}$. We have automatically ordered the factors of $C_{i}$ and ending at $i$ as follows (the other ones do not appear as they play no role in the construction $C_{i+1}$ ) (see Diagram E). Clearly we have

$$
d_{i}=\sum_{u=0}^{r} f_{u}+2 k+l+m,
$$

and we have several possibilities for the dimension $d_{i+1}$ :

(a) If $d_{i+1} \leqslant f_{0}, d_{i+1}$ of the factors $E_{i+1, i_{0}}$ of $C_{i}$ become $E_{i+2, i_{0}}$ in $C_{i+1}$; the remaining $f_{0}-d_{i+1}$ are unchanged. 

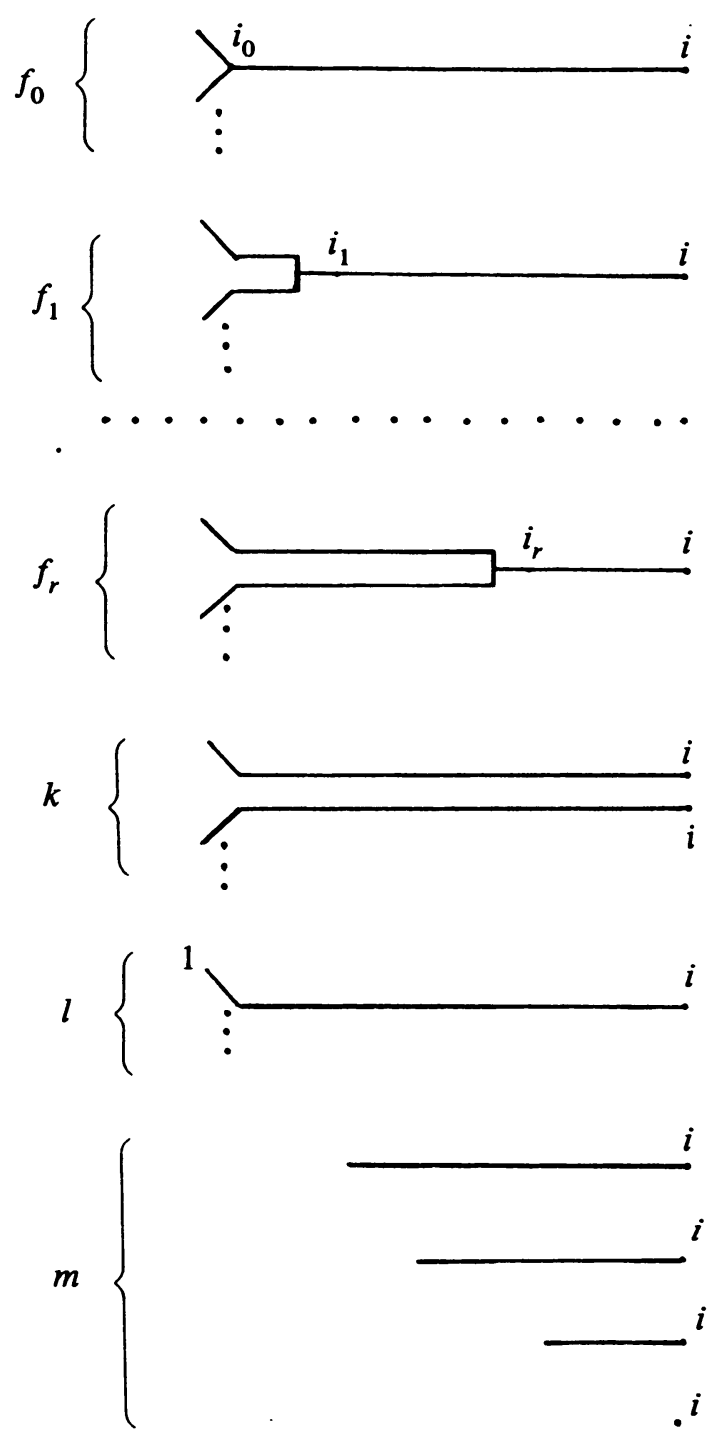

Diagram E

(b) If $d_{i+1}=\sum_{u=0}^{h} f_{u}+t, 0 \leqslant h \leqslant r-1,0<t \leqslant f_{h+1}$, all $f_{u}$ factors $E_{i+1, i}$, $u=0, \ldots, h<r$, of $C_{i}$ become $E_{i+2, i_{u}} ; t$ of the $f_{h+1}$ factors $E_{i+1, i_{h+1}}$ become $E_{i+2, i_{h+1}}$; the remaining $f_{h+1}-t$ are unchanged.

(c) If $d_{i+1}=\sum_{u=0}^{r} f_{u}+t, 0<t \leqslant k$, all $f_{u}$ factors $E_{i+1, i_{u}}, u=0, \ldots, r$, become $E_{i+2, i_{u}}$ and $t$ of the pairs $E_{1 i} \oplus E_{2 i}$ give rise to $t$ factors $E_{i+2, i+1}$; the remaining $k-t$ $E_{1 i} \oplus E_{2 i}$ are unchanged.

(d) If $d_{i+1}=\sum_{u=0}^{r} f_{u}+k+t, 0<t \leqslant l$, all $f_{u}$ factors $E_{i+1, i_{u}}, u=0, \ldots, r$, become $E_{i+2, i}$; all $k$ pairs $E_{1 i} \oplus E_{2 i}$ give rise to $k$ factors $E_{i+2, i+1}$, and $t$ of the $l$ factors $E_{1 i}$ become $E_{1, i+1}$; the remaining $l-t E_{1 i}$ are unchanged.

(e) If $d_{i}=\sum_{u=0}^{r} f_{u}+k+l+t, 0<t \leqslant k$, all $f_{u}$ factors $E_{i+1, i_{u}}$ become $E_{i+2, i_{u}}$; $k-t$ of the pairs $E_{1 i} \oplus E_{2 i}$ become $E_{i+2, i+1}$; the remaining $t$ pairs $E_{1 i} \oplus E_{2 i}$ become $E_{1, i+1} \oplus E_{2, i+1}$; all the $l E_{1 i}$ become $E_{1, i+1}$. 
(f) If $d_{i+1}=\sum_{u=0}^{r} f_{u}+2 k+l+t, 0<t \leqslant m$, all indecomposables containing the vertices 1 or 2 (or both) and end at $i$ are extended to $i+1, t$ of the remaining factors ending at $i$ are extended to $i+1$, the priority being given to the $E_{p i}(p \leqslant i)$ with $p$ small; i.e. if $t=\sum_{p=3}^{s} m_{p}+\tau, s<i, 0<\tau \leqslant m_{s+1} \neq 0$, then all the $E_{p i}$ of $C_{i}$, $p=0, \ldots, s$, become $E_{p, i+1}$ in $C_{i+1}, \tau$ of the $m_{s+1} E_{s+1, i}$ become $E_{s+1, i+1}$ and the remaining are unchanged.

(g) If $d_{i+1}>d_{i}$ all factors of $C_{i}$ ending at $i$ in $C_{i+1}$ are extended to $i+1$, and in $C_{i+1}$ there are also $d_{i+1}-d_{i}$ factors $E_{i+1, i+1}$.

Finally we define $C(d):=C_{n}$. The graphic description of the construction $C_{i+1}$ is very easy once we order the factors of $C_{i}$ ending at $i$ as indicated at the beginning of the discussion. In fact in (a), (b), (f), (g) we drown $d_{i+1}$ vertices $i+1$, starting from the top, each one next to a vertex $i$, if possible, and we join with an orizontal edge $i$ and $i+1$.

EXAMPle. $\left(D_{6}\right.$, eq $), d=\left(\frac{3}{2} ; 4,5,4,1\right)$.

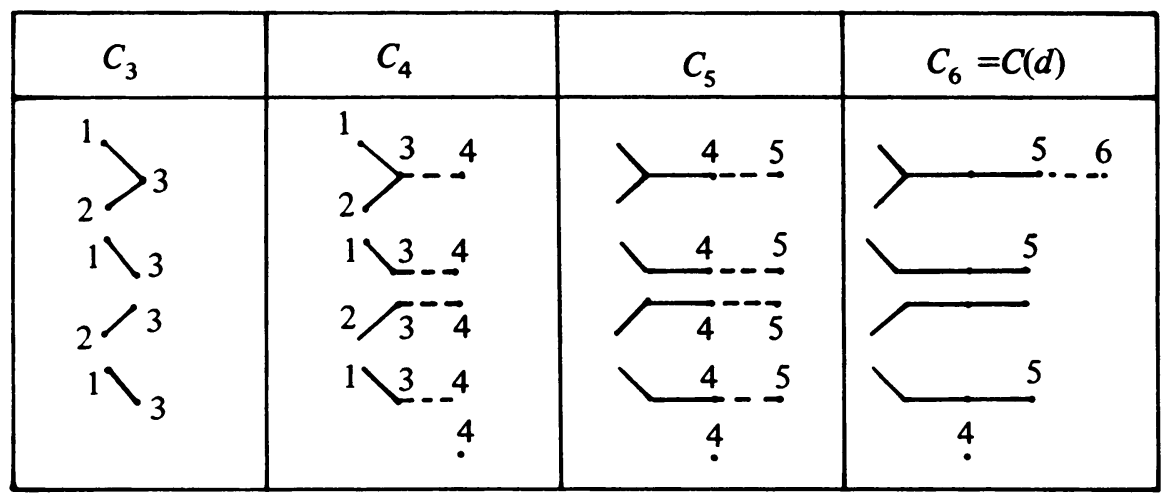

In (c), (e), (d) we distribute the first $\sum_{u=0}^{r} f_{u}$ of the $d_{i+1}$ vertices $i+1$ as before; with the remaining ones we try to extend all factors $E_{1 i}$ and $E_{2 i}$ simultaneously to $i+1$, if possible, but not $E_{p i}$ where $p \geqslant 3$. This implies some of the remaining vertices $i+1$ need to be drawn between a pair $E_{1 i} \oplus E_{2 i}$, creating a factor $E_{i+2, i+1}$.

Example. $\left(D_{6}\right.$, eq $), d=\left({ }_{3}^{4} ; 8,9,6,3\right)$.

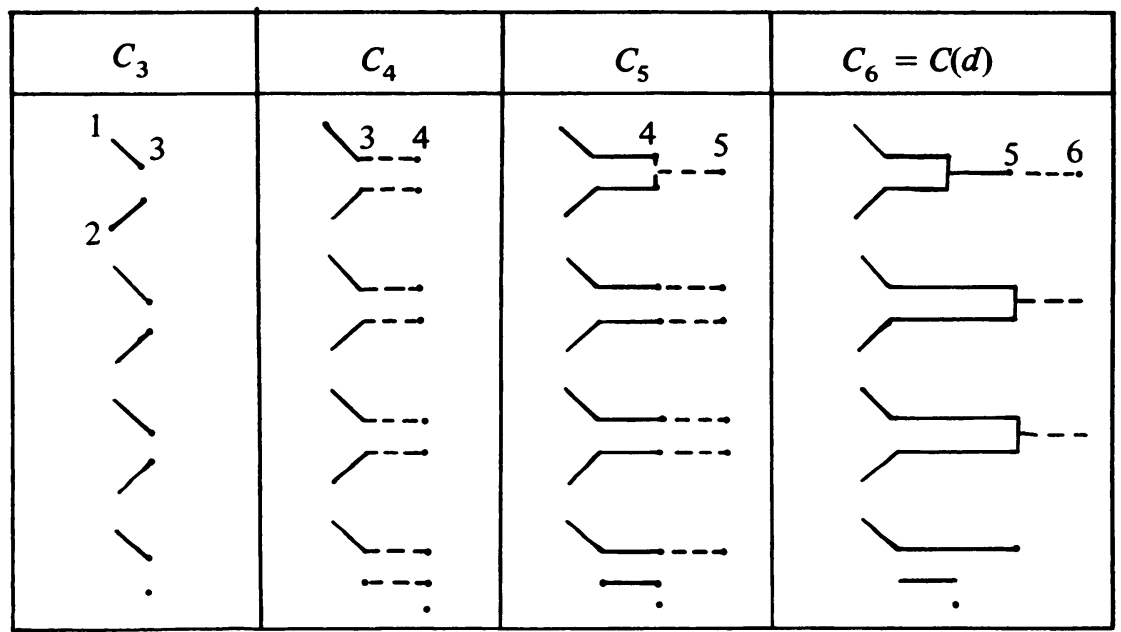


Note that once we have ordered the indecomposables of the construction $C_{i}$ as indicated, the indecomposables of $C_{i+1}$ ending at $i+1$ are automatically ordered to perform $C_{i+2}$. This fact makes the whole construction $C(d)=C_{n}$ very easy to be performed graphically.

LEMMA 3.2. Let $A=C(d)$.

(i) Assume in $A$ we have a factor $E_{z t}, n+1 \geqslant z>t \geqslant 3$. Then no factor $E_{p q}$ can appear in $A$ such that:

(a) $p>q>t, p>z$

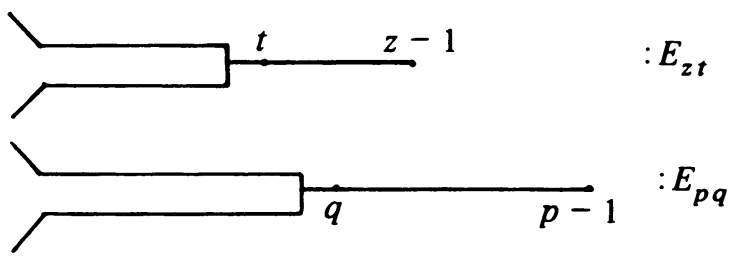

(b) $p \leqslant q$ and either $3 \leqslant p \leqslant t, q \geqslant t$; or $p=z, q \geqslant z$; or $p=1,2, q<t-1$; or $p=1,2, q>z-1$;

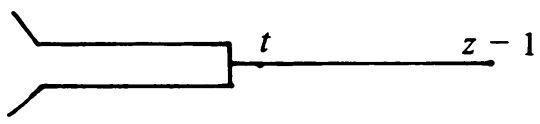

$: E_{z t}$

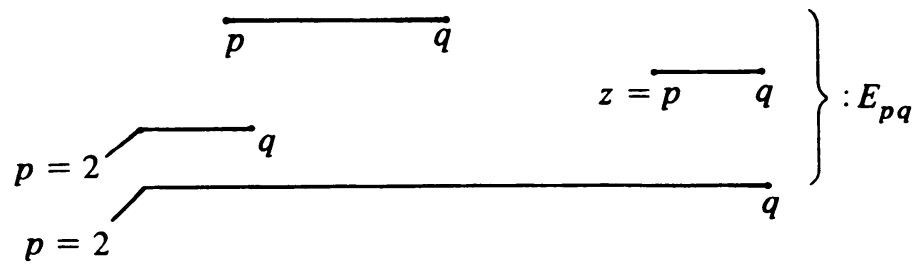

(ii) Assume in $A$ we have a factor $E_{t z}, t \leqslant z, t, z=1, \ldots, n$. Then no factor $E_{p, q}$ can appear in $A$ with $p \leqslant q$ and $p<t, t-1 \leqslant q<z$.

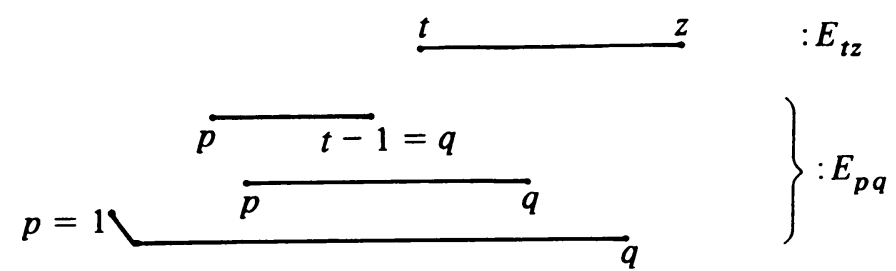

(iii) Assume there is a factor $E_{1 z}$ in $A$. Then no factor $E_{2 q}$ can appear in $A$ with $q \neq z$. 


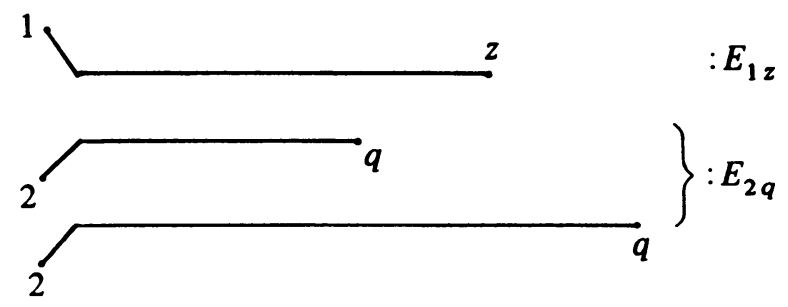

Proof. It is a direct consequence of the construction $C(d)$. If in (i) we assume $E_{z t}$ and $E_{p q}$ in $A, E_{p q}$ as in (a), then we have violated the construction $C(d)$ at the vertex $z$, i.e. $C_{z}$ is not correct. The proof is similar in all the other cases.

Proof of Proposition 3.1. Let $A:=C(d)=C_{n}$. We have to verify that

$$
\begin{aligned}
\operatorname{dim} \text { St } A & =\operatorname{dim} G_{d}-\operatorname{dim} L_{d} \\
& =d_{1}^{2}+\cdots+d_{n}^{2}-\left(d_{1} d_{3}+d_{2} d_{3}+d_{3} d_{4}+\cdots+d_{n-1} d_{n}\right) \\
& =d_{1}\left(d_{1}-d_{3}\right)+d_{2}\left(d_{2}-d_{3}\right)+\cdots+d_{n-1}\left(d_{n-1}-d_{n}\right)+d_{n}^{2} .
\end{aligned}
$$

We proceed by induction and assume

$$
A^{\prime}:=C_{n-1} \in O_{\max }^{d^{\prime}} \subset L_{d^{\prime}}, \quad d^{\prime}=\left(\begin{array}{l}
d_{1} \\
d_{2}
\end{array} ; d_{3}, \ldots, d_{n-1}\right),
$$

i.e.,

$$
\operatorname{dim} \text { St } A^{\prime}=d_{1}\left(d_{1}-d_{3}\right)+d_{2}\left(d_{2}-d_{3}\right)+\cdots+d_{n-2}\left(d_{n-2}-d_{n-1}\right)+d_{n-1}^{2} \text {. }
$$

Then we need only prove

$$
\Delta:=\operatorname{dim} \mathrm{St} A-\operatorname{dim} \mathrm{St} A^{\prime}=d_{n}\left(d_{n}-d_{n-1}\right) .
$$

We compute $\Delta$ using the stabilizer formula (2.2) for both $A$ and $A^{\prime}$. Therefore we need only compare the contribution given to $\operatorname{dim} S t A^{\prime}$ by the factors in $C_{n-1}$ ending at $n-1$ and the contribution given to $\operatorname{dim} \operatorname{St} A$ by the factors in $C_{n}$ ending at $n-1$ and $n$. We read the integers $f_{0}, \ldots, f_{r}, k, l, m$ relative to the construction $C_{n-1}$ and we examine the various possibilities for $C(d)=C_{n}$. If $d_{n}=d_{n-1}$, all the indecomposables ending at $n-1$ in $C_{n-1}$ are extended to $n$. Then from (2.2) we have $\operatorname{dim}$ St $A^{\prime}=\operatorname{dim}$ St $A$ and the claim is proved.

If $d_{n}<d_{n-1}$, i.e. in cases (a) to (f) of the construction, using Table 2 we see that $\operatorname{dim} \mathrm{St} A<\operatorname{dim} \mathrm{St} A^{\prime}$, i.e. $\Delta<0$. Moreover, in (a), (b), (f) the decrease comes from the contribution given in (2.2) from the $d_{n}$ factors ending at $n$, once paired with the $d_{n}-d_{n-1}$ factors which remain unchanged in passing from $C_{n-1}$ to $C_{n}$. Therefore $\Delta=d_{n}\left(d_{n}-d_{n-1}\right)$. In (c) the decrease comes from the $d_{n}$ factors ending at $n$ once paired with those which remain unchanged, and with the $t$ factors $E_{n+1, n}$, i.e.

$$
\begin{aligned}
-\Delta & =[m+l+2(k-t)] d_{n}+t d_{n}=(m+l+2 k-t) d_{n} \\
& =\left(d_{n-1}-d_{n}\right) d_{n} .
\end{aligned}
$$

In (d) the decrease comes from the $d_{n}$ factors ending at $n$ once paired with those which remain unchanged, and with the $k$ factors $E_{n+1, n}$, i.e.

$$
-\Delta=d_{n}[m+l-t]+d_{n} \cdot k=d_{n}[m+l+k-t]=d_{n}\left(d_{n-1}-d_{n}\right) .
$$


In (e) the decrease comes from the $d_{n}$ factors ending at $n$ once paired with $m$ factors which remain unchanged, and with the $k-t$ factors $E_{n+1, n}$, i.e.

$$
-\Delta=d_{n} \cdot m+d_{n}(k-t)=d_{n}(m-k-t)=d_{n}\left(d_{n-1}-d_{n}\right) .
$$

If $d_{n}>d_{n-1}$ then $\operatorname{dimSt} A>\operatorname{dim}$ St $A^{\prime}$ and the increase $\Delta$ comes from the $\left(d_{n}-\right.$ $d_{n-1}$ ) factors $E_{n, n}$ once paired with the $d_{n}$ factors ending at $n$ and coming from $C_{n-1}$, i.e. $\Delta=d_{n}\left(d_{n}-d_{n-1}\right)$. The proposition is now completely proved.

4. The set $I_{d}\left(D_{n}\right.$, eq). Let $d$ as in (*) be a given dimension for the representations of $\left(D_{n}\right.$, eq) such that $d_{h}>0$ for all $h=1, \ldots, n$ and assume $d_{n+1}=0$. We define

$$
I_{d}=I_{d}^{\prime} \cup I_{d}^{\prime \prime},
$$

where

$$
\begin{aligned}
& I_{d}^{\prime}:=\{(i, j): i, j \in[3, n+1], i>j \text { and } \\
& d_{1}<d_{j}<d_{t}, d_{2}<d_{j}<d_{t} \text { for all } t \in[3, j-1], \\
& \left.d_{1}+d_{2}=d_{j}+d_{i}, d_{t}>d_{i} \text { for all } t \in[j, i-1]\right\}, \\
& I_{d}^{\prime \prime}:=\{(i, j): i, j=1,2, \ldots, n, i<j \text { and } \\
& \left.d_{i}=d_{j}<d_{t} \text { for all } t \in[i+1, j-1]\right\}
\end{aligned}
$$

REMARK 4.2. (a) If in (4.1)' we assume $i=n+1$, i.e. $(n+1, j) \in I_{d}^{\prime}$, then the conditions on the $d_{h}$ are

$$
j \in[3, n], \quad d_{1}+d_{2}=d_{j}<d_{t} \text { for all } t \in[3, j-1] .
$$

(b) The conditions defining $(i, j) \in I_{d}^{\prime \prime}$ for $i \neq 1$ (resp. $i \neq 2$ ) are the same as those in [1] for the equioriented graph $\mathscr{A}_{n-1}^{(1)}$ (resp. $\mathscr{A}_{n-1}^{(2)}$ ) and the dimension $d^{(1)}=\left(d_{2}, d_{3}, \ldots, d_{n}\right)\left(\right.$ resp. $\left.\left(d_{1}, d_{3}, \ldots, d_{n}\right)\right)$.

$I_{d}$ is entirely defined in terms of $d$. Its main property here will be to have the same cardinality of the codimension 1 orbits in $L_{d}$ (cf. 4.6 and 4.7).

The following properties are immediate:

LEMMA 4.3. ( $\alpha)$ If $(i, j) \in I_{d}^{\prime}$ then for every $(u, v) \neq(i, j), u \in[i, n+1], v \in[j, i]$, $(u, v) \notin I_{d}^{\prime}$. $I_{d}^{\prime \prime}$

( $\beta)$ If $(i, j) \in I_{d}^{\prime \prime}$ then for every $(u, v) \neq(i, j), u \in[i, j-1], v \in[j, n],(u, v) \notin$

( $\gamma)$ If $(i, j) \in I_{d}^{\prime \prime}$ for every $u \in[j+1, n+1],(u, j) \notin I_{d}^{\prime}$.

Proof. It follows from the fact that if we assume $(i, j)$ and $(u, v)$ are simultaneously in $I_{d}$, the inequalities imposed on $d$ are incompatible. For instance, in $(\alpha)$ if we assume $(i, j),(u, v) \in I_{d}^{\prime}$ and $u \in[i, n+1], v \in[j, i]$, we must have $d_{u}+d_{v}=$ $d_{1}+d_{2}=d_{i}+d_{j}$; moreover, either $d_{j} \geqslant d_{v}$, or $d_{i} \geqslant d_{u}$ must be a strict inequality since $(u, v) \neq(i, j)$, a contradiction.

Next we want to analyze the condition $(i, j) \in I_{d}$ in terms of the indecomposables appearing as factors in $A=C(d)$. 
Interpretation 4.4. Case $(i, j) \in I_{d}^{\prime}$. The discussion is related to the following sets of indices:

$$
\begin{aligned}
& \Theta_{1}:=\Theta_{1}^{i j}=\left\{s: s>i>j, e_{s j}^{A}>0\right\}, \\
& \Theta_{2}:=\Theta_{2}^{i j}=\left\{t: i>t>j, e_{i t}^{A}>0\right\} .
\end{aligned}
$$

If $i \neq n+1$ the conditions $d_{1}<d_{j}<d_{t}, d_{2}<d_{j}<d_{t}, t \in[3, j-1]$ imply that in $A$ every factor containing the vertex 1 or 2 also contains the vertex $j$. The condition $d_{1}+d_{2}>d_{j}$ implies that in $A$ there is at least a factor $E_{s r}, s>j \geqslant r$. The condition $d_{j-1}>d_{j}$ implies there is at least a factor $E_{s j}, s>j$. The conditions $d_{t}>d_{i}>0$, $t \in[j, i-1]$, and $d_{1}+d_{2}=d_{j}+d_{i}$ imply all the factors $E_{s r}, s>r \leqslant j$, are such that $s>i>j$ and in $A$ there is at least a factor $E_{s j}, s>i$. All the factors $E_{w^{\prime} t}$, $w>t>j$, are such that $w \leqslant i$, and in $A$ there is either a factor $E_{i t}, i>t>j$, or a pair $E_{1, i-1} \oplus E_{2, i-1}$. It follows for $(i, j) \in I_{d}^{\prime}$ and $i \neq n+1$ :

$$
\left\{\begin{array}{l}
\Theta_{1}=\left\{s: s>i>j, e_{s j}^{A}>0\right\} \neq \varnothing, \text { and either } \\
\Theta_{2}=\left\{t: i>t>j, e_{i t}^{A}\right\} \neq \varnothing, \text { or } \\
E_{1, i-1} \oplus E_{2, i-1} \text { is a pair of factors in } A .
\end{array}\right.
$$

If $i=n+1$ the condition $d_{1}+d_{2}=d_{j}<d_{t}, t \in[3, j-1]$, again implies all factors of $A$ containing the vertex 1 or 2 also contain the vertex $j$, but in $A$ there is no factor $E_{s r}$ with $s>r \geqslant j$. Then the same argument holds for $\Theta_{2}$ with $i=n+1$. Moreover if $j>3$ and therefore $d_{1}+d_{2}<d_{3}$, the condition $d_{t}>d_{j}, t \in[3, j-1]$, implies in $A$ there is at least a factor $E_{3, j-1}$; if $j=3$ we will conventionally consider $E_{3, j-1}$ as the zero representation.

It follows for $(n+1, j) \in I_{d}^{\prime}$ :

$$
\left\{\begin{array}{l}
\Theta_{1}=\varnothing, E_{3, j-1} \text { is a factor in } A, \text { and either } \\
\Theta_{2}=\left\{t: t>j, e_{n+1, t}^{A}>0\right\} \neq \varnothing, \text { or } \\
E_{1 n} \oplus E_{2 n} \text { is a pair of factors in } A .
\end{array}\right.
$$

$(4.4)_{1}$ and $(4.4)_{2}$ will classify the possible different cases treated in Table I of $\S 5$.

Corollary 4.4. If $(i, j) \in I_{d}^{\prime}$ then $\varphi_{i j}^{A}$ is an isomorphism.

Proof. The condition $d_{1}+d_{2}=d_{i}+d_{j}$ if $i \neq n+1$, or $d_{1}+d_{2}=d_{j}$ if $i=n+1$ says $\varphi_{i j}^{A}$ is a linear map between vector spaces of the same dimension. But we also deduce that $\operatorname{ker} \varphi_{i j}^{A}=0$, since, according to the Interpretation 4.4, no indecomposable contributing to $\operatorname{ker} \varphi_{i j}^{A}$ is a factor in $A$.

Interpretation 4.5. Case $(i, j) \in I_{d}^{\prime \prime}$. The discussion is now related to the following sets:

$$
\begin{aligned}
& \Lambda_{0}:=\Lambda_{6}^{i j}=\left\{(r, s): 3 \leqslant r \leqslant i<j \leqslant s \leqslant n, e_{r s}^{A}>0\right\}, \\
& \Lambda_{1}:=\Lambda_{1}^{i j}=\left\{(s, r): s>r>j, e_{s r}^{A}>0\right\}, \\
& \Lambda_{2}:=\Lambda_{2}^{i j}=\left\{k: k \geqslant j, e_{1 k}^{A}>0, e_{2 k}^{A}>0\right\}, \\
& \Lambda_{3}:=\Lambda_{3}^{i j}=\left\{k: k \geqslant j, e_{1 k}^{A}>0, e_{2 k}^{A}=0\right\},
\end{aligned}
$$




$$
\begin{aligned}
& \Lambda_{4}:=\Lambda_{4}^{i j}=\left\{(s, r): s>j>i \geqslant r, e_{s r}^{A}>0\right\}, \\
& \Lambda_{5}:=\Lambda_{\xi}^{i j}=\left\{(s, r): s>j \geqslant r \geqslant 3, e_{s r}^{A}>0\right\} .
\end{aligned}
$$

Consider $i \neq 1,2$. Then we can assume $d_{1} \geqslant d_{2}$ (cf. construction $C(d)$ in $\S 3$ ). The condition $d_{i}=d_{j}<d_{t}, t \in[i+1, j-1]$, implies that in $A$ there is at least a factor $E_{i+1, j-1}$ (we read it as the zero representation if $j=i+1$ ). Every factor which contains the vertex $i$ also contains $j$ and conversely; moreover the assumption $d_{h}>0$ for all $h$ implies that in $A$ there are factors passing through $i$ and $j$. Therefore we have the following possibilities: either $\Lambda^{i j} \neq \varnothing$ or all the factors of $A$ containing $i$ and $j$ also contain at least one of the vertices 1,2 . Moreover if $E_{s r}, s>r$, is such a factor then either $s>j>i \geqslant r$ or $s>r>j$. The previous discussion implies that if $i \neq 1,2$ then

$$
\left\{\begin{array}{l}
\text { either } \Lambda_{\gamma}^{i j} \neq \varnothing \text { or } \\
\Lambda_{\tau}^{i j} \neq \varnothing \text { for at least a } \tau, \tau=1,2,3,4 .
\end{array}\right.
$$

Consider $i=1,2$. For the symmetry of $\left(D_{n}\right.$, eq) we may assume $i=1$, i.e. $(1, j) \in I_{d}^{\prime \prime}$, $j \in[3, n]$, but then we have to consider $d_{1} \gtreqless d_{2}$. The condition $d_{1}=d_{j}<d_{t}$, $t \in[3, j-1]$, implies every factor of $A$ containing 1 also contains $j$ and conversely, and such factors exist in $A$ (as $d_{h}>0$ for all $h$ ). If $d_{2} \geqslant d_{1}$ each factor containing 1 also contains 2 and $j$. If $d_{2}<d_{1}$ in $A$ there is no factor $E_{2 h}$.

We have the following possibilities:

$(4.5)_{2} d_{2}>d_{1}, \Lambda_{5}^{i j} \neq \varnothing$ and in $A$ there is at least a factor $E_{2, j-1}$ if $j>3$ or $E_{22}$ if $j=3$.

(4.5) ${ }_{3} d_{1}=d_{2}$, then $(s, j) \in \Lambda_{\xi}^{i j}$ for some $s$, and $\Lambda_{3}^{i j}=\varnothing$.

$(4.5)_{4} d_{2}<d_{1}, \Lambda_{3}^{i j} \neq \varnothing,(s, j) \in \Lambda_{5}^{i j}$ for some $s$ and $s>k$ for all $k \in \Lambda_{5}^{i j}$. We will use $(4.5)_{1}-(4.5)_{4}$ to classify all possible cases listed in Table II of $\S 5$.

COROllaRY 4.5. If $(i, j) \in I_{d}^{\prime \prime}$ then $\varphi_{i j}^{a}$ is an isomorphism.

Proposition 4.6. The cardinality of $I_{d}$ is $\left|I_{d}\right|=n-\tau$, where $n$ is the number of vertices of the Dynkin diagram $D_{n}$ and $\tau$ is the number of nonisomorphic indecomposable factors of $A=C(d)$.

Proof. As usual, we assume $d_{2} \leqslant d_{1}$. Let

$$
d^{\prime}=\left(\begin{array}{l}
d_{1} \\
d_{2}
\end{array} d_{3}, \ldots, d_{n-1}\right)
$$

and consider it as a dimension for the subdiagram of $D_{n}$ obtained by erasing the vertex $n$ (and with the induced orientation). By induction assume $\left|I_{d^{\prime}}\right|=n-1-\tau^{\prime}$, where $\tau^{\prime}$ is the number of nonisomorphic factors in $A^{\prime}=C\left(d^{\prime}\right)=C_{n-1}(d)$.

The case $d_{2}<d_{1}$. We may have either $\left|I_{d}\right|=\left|I_{d^{\prime}}\right|$ or $\left|I_{d}\right|=\left|I_{d^{\prime}}\right|+1$, since by adding the vertex $n$ and passing from $d^{\prime}$ to $d$ we can create at most a new pair $(i, j) \in I_{d}$ with $i$ or $j$ equal to $n$ (cf. Lemma 4.3). We now use the same notation as in $\$ 3$ and in the proof of Proposition 3.1, i.e. the multiplicities $f_{0}, \ldots, f_{r}, k, l, m=$ $\sum_{i=3}^{n-1} m_{i}$ are referred to the construction $C_{n-1}(d)$. If $k \neq 0$ in $A=C(d)$ there is no factor $E_{1, t}$ or $E_{2 t}$ with $t<n-1$ (cf. Lemma 3.2(iii)), and the condition $d_{2}<d_{1}$ implies $l \neq 0$. It follows that in any case we can only have $\tau=\tau^{\prime}$ or $\tau=\tau^{\prime}+1$. Using the inductive assumption we need only prove $\tau=\tau^{\prime}$ if and only if $\left|I_{d}\right|=\left|I_{d^{\prime}}\right|$ +1 . 
The definition of $C(d)$ implies we have $\tau=\tau^{\prime}$ if and only if $0<d_{n} \leqslant d_{n-1}$ and $d_{n}$ satisfies one of the following equalities:

$$
\begin{aligned}
& d_{n}=\sum_{u=0}^{h} f_{u}, \quad h \leqslant r \\
& d_{n}=\sum_{u=0}^{r} f_{u}+k \\
& d_{n}=\sum_{u=0}^{r} f_{u}+k+l \\
& d_{n}=\sum_{u=0}^{r} f_{u}+2 k+l ; \\
& d_{n}=\sum_{u=0}^{r} f_{u}+2 k+l+\sum_{t=3}^{h} m_{t}, \quad h \leqslant n-1, m_{h} \neq 0 .
\end{aligned}
$$

On the other hand if $d_{n}>d_{n-1}$, no pair $(i, j) \in I_{d}$ is such that either $i$ or $j$ equals $n$. If $d_{n}=d_{n-1}$ then $\tau=\tau^{\prime}$ (special case of (4) when $h=n-1$ ), and obviously we have $(n-1, n) \in I_{d}$ and conversely. Therefore we are only left with the case $d_{n}<d_{n-1}$. Note that (1) disappears if the sequence $\left\{f_{0}, \ldots, f_{r}\right\}$ is empty. Moreover (1)-(4) need not be distinct; in fact, for example, if $k=0$ and $h=r$, (1) and (2) coincide; if $k=l=0$ and $m \neq 0$ then (2), (2') and (3) coincide. If this happens we only consider the case corresponding to the equality of higher index.

Assume $\tau=\tau^{\prime}$. Then $\left(I_{d}\right)=\left|I_{d^{\prime}}\right|+1$. In fact:

if (1) holds, $\left(n, i_{h}\right) \in I_{d}^{\prime}$;

if (2) holds, $(2, n) \in I_{d}^{\prime \prime}$;

if $\left(2^{\prime}\right)$ holds, $(1, n) \in I_{d}^{\prime \prime}$;

if (3) holds $(i, n) \in I_{d}^{\prime \prime}$, if $i+1>3$, or $(n+1, n) \in I_{d}^{\prime}$, if $i+1=3$ and $i+1$ is always the minimum index such that $m_{i+1}>0$;

if (4) holds, $(i, n) \in I_{d}^{\prime \prime}$, with $h<i+1 \leqslant n+1$ and $i+1$ is the minimum index such that $m_{i+1}>0$, which follows from the fact that

$$
d_{n-1}=\sum_{u=0}^{r} f_{u}+2 k+l+m>d_{n}
$$

and Lemma 3.2. The converse is also true, i.e. if we have a pair $(i, j) \in I_{d}$ and either $i$ or $j$ are equal to $n$, then $\tau=\tau^{\prime}$ as an easy consequence of Interpretations 4.4 and 4.5 of $(i, j) \in I_{d}$ in terms of the canonical construction.

The case $d_{1}=d_{2}$. Adding the vertex $n$ we may only have $\left|I_{d}\right|=\left|I_{d^{\prime}}\right|+\rho, \rho=0,1,2$, and the equality holds with $\rho=2$ if and only if $d_{n}=d_{1}=d_{2}$ and $d_{t}>d_{n}$, $t \in[3, n-1]$. On the other hand we also have $\tau=\tau^{\prime}+\delta, \delta=0, \pm 1$, and we have $\delta=-1$ if and only if $d_{n}<d_{n-1}, k \neq 0$, and $d_{n}=\sum_{u=0}^{r} f_{u}+k$, but $d_{1}=d_{2}$ now implies $d_{n}=d_{1}=d_{2}$ and $d_{t}>d_{n}, t \in[3, n-1]$. Therefore also in this case, using the inductive assumption, we need only prove $\tau=\tau^{\prime}$ if and only if $\left|I_{d}\right|=\left|I_{d^{\prime}}\right|+1$. The discussion now is the same as in the case $d_{2}<d_{1}$, we need only erase (2), (2') and the corresponding discussion if $k \neq 0$. 
The importance of this proposition comes from the following results of Happel [9] and Sato and Kimura [13] (cf. also §6).

Proposition 4.7. (a) $n-\tau$ equals $\operatorname{dim} L_{d} / \Pi \operatorname{SL}\left(V_{i}\right)$,

(b) $n-\tau$ equals the number of codim. 1 orbits in $L_{d}$.

5. The set $\mathcal{O}_{1}$ of codim. 1 orbits in $L_{d}\left(D_{n}\right.$, eq).

THEOREM 5.1. There exists a unique bijection $\psi: I_{d} \rightarrow \mathcal{O}_{1}, \psi(i, j)=O_{i j}, B_{i j} \in O_{i j} a$ representative such that

(a) $N_{i j}^{B_{i j}}=N_{i j}^{A}-1$,

(b) $N_{u v^{\prime}}^{B_{i j}}=N_{u u^{\prime}}^{A}$ for all $(u, v) \in I_{d},(u, v) \neq(i, j)$.

Proof. We need only construct an injective map $\psi$ satisfying (a) and (b). Then $\psi$ is automatically bijective as a consequence of Propositions 4.6 and 4.7.

The definition of $\psi$ is contained in Table I if $(i, j) \in I_{d}^{\prime}$ and Table II if $(i, j) \in I_{d}^{\prime \prime}$, and is inspired by the fact that the rank parameters parametrize the orbits and by the following consideration: $(i, j) \in I_{d}$ implies $\varphi_{i j}^{A}$ is an isomorphism (cf. Corollaries $4.4,4.5)$. Then if $B_{i j}$ must be a representative for a codim. 1 orbit satisfying (a), we have to produce a "damage" to $\varphi_{i j}^{A}$ and make it as small as possible. Now the rank parameters for a representation satisfy the inequalities of Proposition 1.4 , therefore from the "damage" produced to $\varphi_{i j}^{A}$ it may follow a "damage" to $\varphi_{u v}^{A}$ for other pairs $(u, v)$ and in any case we have to try to minimize their number. We explain how to read the tables. In column 1 are listed the various cases which can occur when $(i, j) \in I_{d}$ according to Interpretations 4.4, 4.5. In column 3 we define (graphically, for convenience) a representation $A_{i j}^{\prime} \neq 0$ for a suitable choice of the indices listed in column 2. $A_{i j}^{\prime}$ is the maximal orbit corresponding to its dimension and consists of at most three factors. Clearly we have the decomposition

$$
A=A_{i j}^{\prime} \oplus \bar{A}
$$

In column 4 we define a representation $B_{i j}^{\prime}$ such that $B_{i j}^{\prime} \neq A_{i j}^{\prime}$ and $\operatorname{dim} B_{i j}^{\prime}=\operatorname{dim} A_{i j}^{\prime}$. $B_{i j}^{\prime}$ is constructed from $A_{i j}^{\prime}$ via a suitable degeneration (a violation to the canonical construction either at vertex $i$ or vertex $j$ ).

We define the map $\psi$ on the pair $(i, j) \in I_{d}$ via the following representative:

$$
B_{i j}=B_{i j}^{\prime} \oplus \bar{A}, \quad \psi(i, j)=O_{i j} \ni B_{i j}
$$

and we need to check that:

$$
\operatorname{codim}_{L_{d}} \bar{O}_{B_{\imath}}=1
$$

The pair $(i, j) \in I_{d}$ determines the decomposition (5.2) of $A$, i.e. the representation $A_{i j}^{\prime}$. This claim is trivial in Table I; in Table II the ambiguity comes from the fact that in some cases we have to deal in column 2 with sets of pairs of indices, but the claim follows from Lemma 3.2 applied to the factors of $A_{i j}^{\prime}$. Consider, for example, case 1 of Table II and assume, by contradiction, $(r, s) \in \Lambda_{0},\left(r^{\prime}, s^{\prime}\right) \in \Lambda_{0}$, $(r, s) \neq\left(r^{\prime}, s^{\prime}\right)$ and $s-r=s^{\prime}-r^{\prime}$ the least possible. Then in $A$ there are simultaneously two factors $E_{r s}, E_{r^{\prime} s^{\prime}}$, with $r^{\prime}<r<s^{\prime}<s$, a contradiction to Lemma 3.2(ii). For all other cases we always use Lemma 3.2(i). 


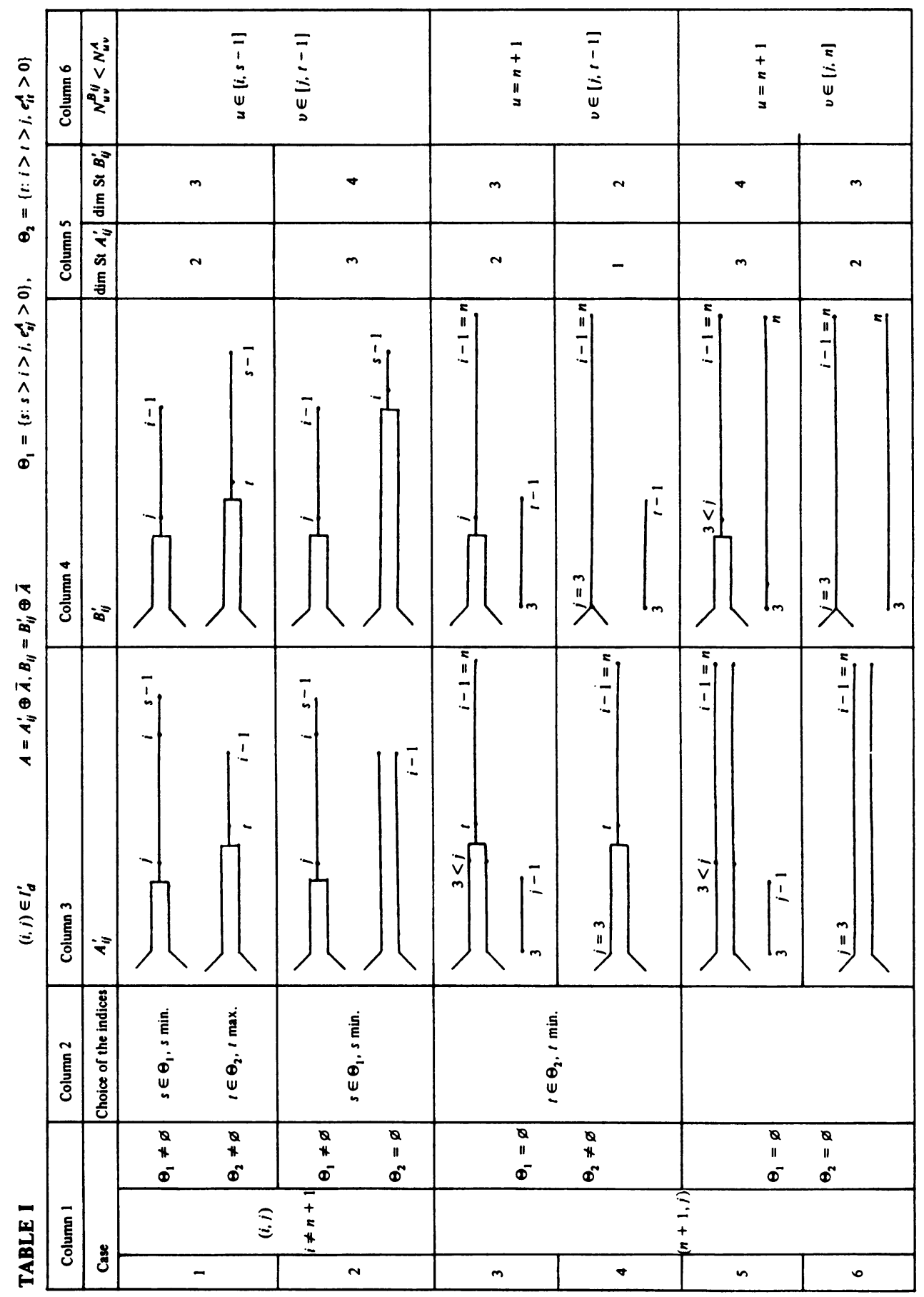




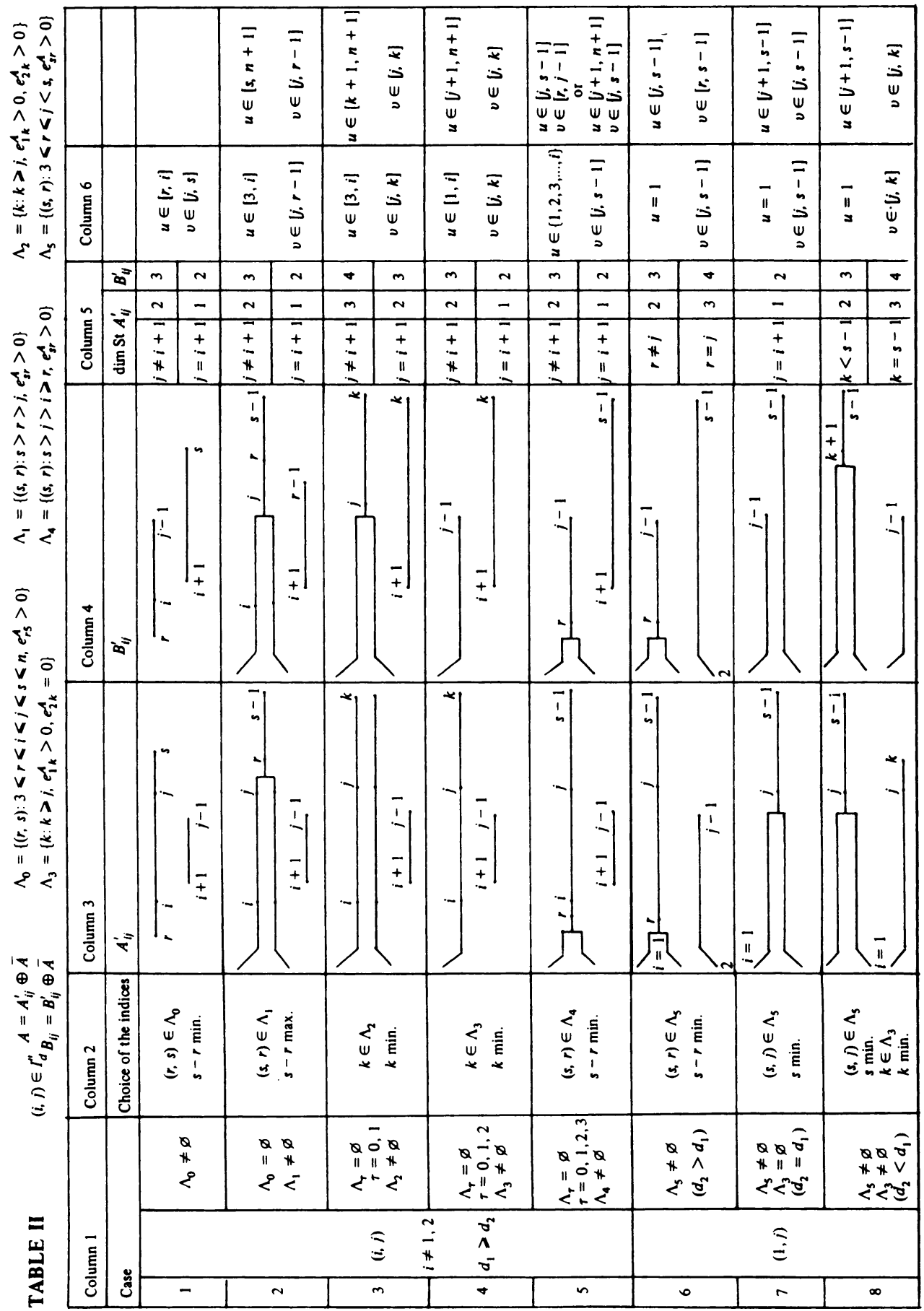


In Table I we have collected all possible cases which can occur when $(i, j) \in I_{d}^{\prime}$. If $(i, j) \in I_{d}^{\prime \prime}$ and $i \neq 1,2$ in Table II, we have not explicitly drawn, for economy, the "special case" $j=i+1$; if so we mean that the factor $E_{i+1, j-1}$ in $A_{i j}^{\prime}$ is the zero representation (i.e. we erase this factor in column 3 ) and in column 4 the representation $B_{i j}^{\prime}$ is formally unchanged (but $i+1=j$ !). Moreover, we have assumed $d_{1} \geqslant d_{2}$, as we can read the case $d_{1}<d_{2}$ using the symmetry of $\left(D_{n}\right.$, eq) with respect to the permutation of the vertices 1 and 2 . The only representation $A_{i j}^{\prime}$ which is not invariant under such a permutation is the one listed in case 4; therefore if $d_{1}<d_{2}$ we must replace the set $\Lambda_{3}$ with $\Lambda_{3}^{\prime}=\left\{k: k \geqslant j, e_{2 k}^{A}>0, e_{1 k}^{A}=0\right\}$, the factor $E_{1 k}$ with $E_{2 k}$ in column 3 and $E_{1, j-1}$ with $E_{2, j-1}$ in column 4 . In Table II we have only considered the case $(1, j) \in I_{d}^{\prime \prime}$, but we have assumed $d_{1} \gtreqless d_{2}$; therefore the situation for $(2, j) \in I_{d}$ can be read from the previous one by permuting the indices 1 and 2 . If $(1, j) \in I_{d}^{\prime \prime}$ and $d_{2}>d_{1}$, we have the "special case" $j=r$ in column 3 . Then in column 4 we replace the factor $E_{j r}$ with the pair $E_{1, j-1} \oplus E_{2, j-1}$ if $j \neq 3$, or with $E_{11} \oplus E_{22}$ if $j=3$. If $(1,3) \in I_{d}^{\prime \prime}$ and $d_{1}=d_{2}$ in column 4 , we replace $E_{1, j-1}$ with $E_{11}$. If $(1, j) \in I_{d}^{\prime \prime}$ and $d_{2}<d_{1}$ we may have $k=s-1$; in this case in column 4 we replace the factor $E_{s, k+1}$ with the pair $E_{1, s-1} \oplus E_{2, s-1}$.

In order to have $\psi: I_{d} \rightarrow \mathcal{O}_{1}$ well defined we have to prove (5.4) on the codimension. We use the following procedure. We have $\operatorname{codim}_{L_{d}} \bar{O}_{i j}=\operatorname{dim} \operatorname{St} B_{i j}-\operatorname{dim} \mathrm{St} A$. We use the stabilizer formula (2.4) and the decompositions (5.2) and (5.3), respectively, for $A$ and $B_{i j}$; we get

$$
\begin{aligned}
\operatorname{codim}_{L_{d}} \bar{O}_{i j} & =\operatorname{dim} \text { St } B_{i j}^{\prime}-\operatorname{dimSt} A_{i j}^{\prime}+\left[B_{i j}^{\prime}, \bar{A}\right]-\left[A_{i j}^{\prime}, \bar{A}\right] \\
& =\operatorname{dimSt} B_{i j}^{\prime}-\operatorname{dimSt} A_{i j}^{\prime}+\sum_{p, q} e_{p q}^{\bar{A}}\left\{\left[B_{i j}^{\prime}, E_{p q}\right]-\left[A_{i j}^{\prime}, E_{p q}\right]\right\} .
\end{aligned}
$$

In column 5 of Tables I or II we have listed the values of $\operatorname{dim}$ St $A_{i j}^{\prime}$ and $\operatorname{dim}$ St $B_{i j}^{\prime}$ (this computation is immediate from Table 2 and (2.3), since we know explicitly the factors in $A_{i j}^{\prime}$ and $B_{i j}^{\prime}$ ). From column 5 we read

$$
\operatorname{dim} \text { St } B_{i j}^{\prime}-\operatorname{dim} \mathrm{St} A_{i j}^{\prime}=1 \text {. }
$$

It follows that

$$
\operatorname{codim}_{L_{d}} \bar{O}_{i j}=1+\sum_{p, q} e_{p q}^{\bar{A}}\left\{\left[B_{i j}^{\prime}, E_{p q}\right]-\left[A_{i j}^{\prime}, E_{p q}\right]\right\}
$$

and we need only verify that

$$
\sum_{p . q} e_{p q}^{\bar{A}}\left\{\left[B_{i j}^{\prime}, E_{p q}\right]-\left[A_{i j}^{\prime}, E_{p q}\right]\right\}=0
$$

(5.5) has been checked case by case using the following argument. In (5.5) we have no contribution whenever $E_{p q}$ is such that $e_{p q}^{\bar{A}}=0$. In view of this we take the list of the indecomposables $E_{p, q}, p \gtreqless q$, of $D_{n}$ given in $\$ 1$ and erase the ones which are incompatible with the interpretation of $(i, j) \in I_{d}$ in 4.4 or 4.5 , the definition of $A_{i j}^{\prime}$ (i.e. the indices in column 2) and Lemma 3.2 applied to the factors of $A_{i j}^{\prime}$ (in this case in fact we know a priori that $e_{p q}^{\bar{A}}=0$ ); then we draw the list of the remaining ones (specifying the mutual position of the indices $p, q$ with respect to the given $i, j$ ) and check directly, using Table 2 , that $\left[B_{i j}^{\prime}, E_{p q}\right]-\left[A_{i j}^{\prime}, E_{p q}\right]=0$. 
The proof of (5.5) is therefore just routine and is very boring due to the large number of cases. We explicitly give just one case, and we choose case 1 of Table II because the orbit $O_{i j}$ is constructed in the same way as for an equioriented Dynkin diagram of type $\mathscr{A}_{m}$ (cf. Remark $4.2(\mathrm{~b})$ ) and [1]; nevertheless we must prove (5.4) and, therefore, (5.5) as in $D_{n}$ there are indecomposable representations which are not representations for any subdiagram $\mathscr{A}_{m_{-}}$of $D_{n}$. Case 1 . Table II: the list of the $E_{p q}$ for which we do not know a priori that $e_{p q}^{\bar{A}}=0$ is the following diagram (Diagram F). We immediately read, using Table 2 , that

$$
\left[E_{r, j-1} \oplus E_{i+1, s}, E_{p q}\right]-\left[E_{r s} \oplus E_{i+1, j-1}, E_{p q}\right]=0
$$

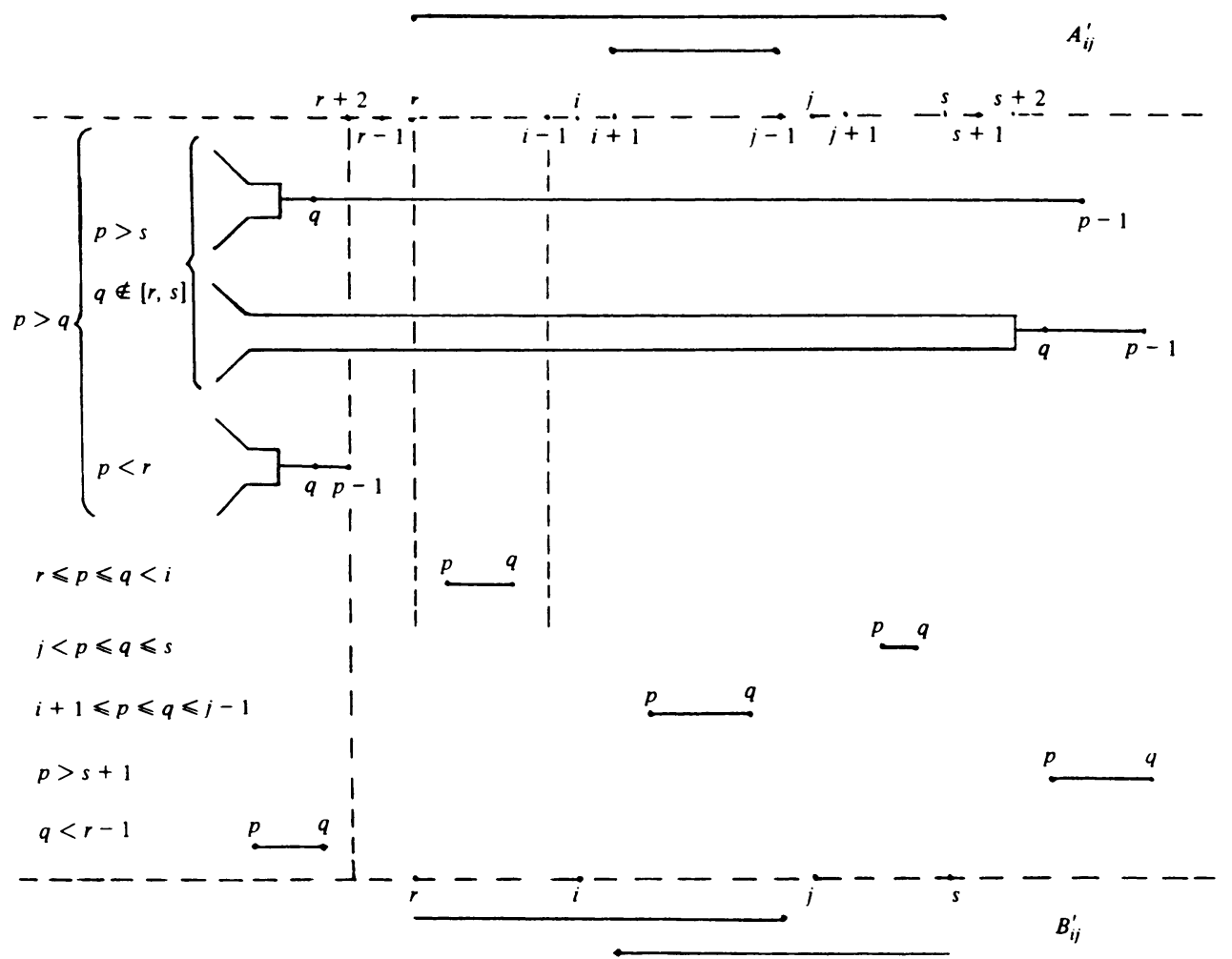

\section{Diagram $\mathrm{F}$}

To end the description of the tables we explain column 6. For $B_{i j}$ given by (5.3) we have $N_{u v}^{B_{i}} \leqslant N_{u v}^{A}$ and strict inequality holds for at least a pair $(u, v)$, since $B_{i j} \in L_{d}$ and $A$ is a representative of the maximal orbit (cf. Proposition 1.5). Column 6 lists all pairs for which strict inequality holds. These pairs are easily computed once we observe that ranks are additive; therefore we need only compare rank parameters for $A_{i j}^{\prime}$ and $B_{i j}^{\prime}$, which are explicit, using definitions $\left(1^{\prime}\right),\left(2^{\prime}\right),\left(3^{\prime}\right)$. We always get $N_{u v}^{B_{i j}}=N_{u v}^{A}-1$, except for $u>v$ and $u \in[j+1, s-1], v \in[j, s-$ 1] of case 5, Table II, where $N_{u v}^{B_{i j}}=N_{u v}^{A}-2$.

Note that if we assume $A_{i j}^{\prime}$ and $B_{i j}^{\prime}$ as in columns 3 and 4 , but with an arbitrary choice of the indices in the sets $\Lambda_{\tau}$ or $\Theta_{\rho}$, column 6 remains unchanged; suitable 
choice of the indices of column 2 minimizes the intervals listed in column 6, i.e. the number of rank parameters which have decreased their value passing from $A$ to $B_{i j}$.

Injectivity of $\psi$. We must show that $(i, j),\left(i^{\prime}, j^{\prime}\right) \in I_{d},(i, j) \neq\left(i^{\prime}, j^{\prime}\right)$ imply $\psi(i, j) \neq \psi\left(i^{\prime}, j^{\prime}\right)$ (i.e. $\left.B_{i j} \neq B_{i^{\prime} j^{\prime}}\right)$. Consider simultaneously the decompositions (5.2) for the pairs $(i, j)$ and $\left(i^{\prime}, j^{\prime}\right)$ :

$$
A=A_{i j}^{\prime} \oplus \bar{A}, \quad A=A_{i^{\prime} j^{\prime}}^{\prime} \oplus \tilde{A},
$$

and the corresponding degenerations:

$$
B_{i j}=B_{i j}^{\prime} \oplus \overline{A,} \quad B_{i^{\prime} j^{\prime}}=B_{i^{\prime} j^{\prime}}^{\prime} \oplus \tilde{A} .
$$

If $A_{i j}^{\prime} \neq A_{i^{\prime} j^{\prime}}^{\prime}$ in $A_{i j}^{\prime}$ there is at least a factor $E_{z w}$ which is not a factor of $A_{i^{\prime} j^{\prime}}^{\prime}$ and therefore is a factor in $\tilde{A}$ and $B_{i^{\prime}, j^{\prime}}$. But $E_{z w^{\prime}}$ has been destroyed by the construction $\psi(i, j)$, i.e. $E_{z w}$ is not a factor in $B_{i j}^{\prime}$. If $A_{i j}^{\prime}=A_{i^{\prime} j^{\prime}}^{\prime}$ (it can happen even if $(i, j) \neq\left(i^{\prime}, j^{\prime}\right)$, cf. case 7 in Table II), then $B_{i j}^{\prime} \neq B_{i^{\prime} j^{\prime}}^{\prime}$, and $\bar{A}=\tilde{A}$. In any case $B_{i j} \neq B_{i^{\prime} j^{\prime}}$ and the injectivity of $\psi$ is proved.

Proof of (a), (b). (a) is trivial since the pair $(i, j)$ is in the list of column 6 and $N_{i j}^{B_{i \prime}}=N_{i j}^{A}-1$. To prove (b) assume $(i, j) \in I_{d}, N_{u v}^{B_{i j}}<N_{u i^{\prime}}^{A}$ for $(u, v) \neq(i, j)$ and $(u, v) \in I_{d}$. Then $\psi(i, j)$ is defined via the representative $B_{i j}=B_{i j}^{\prime} \oplus \bar{A}$, for $B_{i j}^{\prime}$ listed in one of the cases in Tables I, II(column 4), and $(u, v)$ is a pair listed in column 6 for the same case.

But we claim these conditions are incompatible. For Table I the claim follows directly from Lemma 4.3( $\alpha)$. The discussion for Table II is more delicate, and from Lemma $4.3(\beta),(\gamma)$ the claim follows only if $u$ or $v$ is one of the indices $i, j$, and for $u<v$ in cases $6,7,8$.

For the remaining cases of Table II we proceed as follows: we consider the decomposition $A=A_{u v}^{\prime} \oplus \tilde{A}$ coming from the construction of the map $\psi$ on the pair $(u, v) \in I_{d}$ and we show that at least a factor in $A_{u v}^{\prime}$ contradicts the construction of $\psi$ on the pair $(i, j)$.

Case 1 . We have $u \in[r, i], v \in[j, s], u \neq i, v \neq j \geqslant 3$; then $(u, v) \in I_{d}^{\prime \prime}, 3 \leqslant r \leqslant$ $u<i<j<v \leqslant s$. It follows from the construction $\psi$ on $(u, v)$ (cf. Table II, cases $1-5$ and read $(u, v)$ instead of $(i, j))$ that $A=A_{u v}^{\prime} \oplus \tilde{A}=E_{u+1, v-1} \oplus \cdots$ and $r<u+1 \leqslant i<j \leqslant v-1<s$, a contradiction to the choice of the indices $(r, s) \in$ $\Lambda^{\prime} 6$ in column 2 .

Case 2. If $u \in[3, i], v \in[j, r-1]$ we repeat the argument of Case 1 and get $A=A_{u v}^{\prime} \oplus \bar{A}=E_{u+1, v-1} \oplus \cdots, 3<u+1 \leqslant i<j<v$, a contradiction to the assumption $\Lambda_{\gamma}^{i}=\varnothing$. If $u \in[s, n+1], v \in[j, r-1], v \neq j$, then $(u, v) \in I_{d}^{\prime}$ and $v>j>3$ and the construction $\psi$ on the pair $(u, v)$ is listed in Table I, cases 1-5. Therefore either we have

$$
A=A_{u v}^{\prime} \oplus \tilde{A}=E_{w v} \oplus \cdots, \quad v<r, \quad w-1 \geqslant u \geqslant s,
$$

i.e. a contradiction to the choice of the indices $(s, r) \in \Lambda_{1}^{i j}$ in column 2 , or

$$
A=A_{u v}^{\prime} \oplus \tilde{A}=E_{3, v-1} \oplus \cdots, \quad v-1 \geqslant j>3,
$$

i.e. a contradiction to the assumption $\Lambda_{0}^{i j}=\varnothing$.

Case 3. If $u \in[3, i], v \in[j, k], u \neq i, v>j>3$ we have

$$
A=A_{u v}^{\prime} \oplus \tilde{A}=E_{u+1, v-1} \oplus \cdots, \quad 3<u+1 \leqslant i<j \leqslant v-1,
$$


i.e. a contradiction to the assumption $\Lambda_{0}^{i j}=\varnothing$. If $u=n+1, v \in[j, k], v \neq j>3$, we have

$$
A=A_{u v}^{\prime} \oplus \tilde{A}=E_{3, v-1} \oplus \cdots, \quad v-1 \geqslant j>i \geqslant 3,
$$

i.e. a contradiction to the assumption $\Lambda_{0}^{i j}=\varnothing$. If $u \in[k+1, n], v \in[j+1, n]$, either we have

$$
A=A_{u v}^{\prime} \oplus \tilde{A}=E_{w v} \oplus \cdots, \quad w>v \geqslant j+1, \quad w>v,
$$

a contradiction to the assumption $\Lambda_{1}^{i j}=\varnothing$, or

$$
A=A_{u v}^{\prime} \oplus \tilde{A}=E_{1, v-1} \oplus E_{2 v-1} \oplus \cdots, \quad v-1<k,
$$

a contradiction to the choice of the index $k$ in $\Lambda_{2}^{i j}$.

We leave the other cases to the reader. Note that the map $\psi$ gives for any dimension $d$ the codim. 1 orbits through the factors of their decomposition, as we know the decomposition of $A=C(d) \in O_{\max }$.

EXAMPLE.

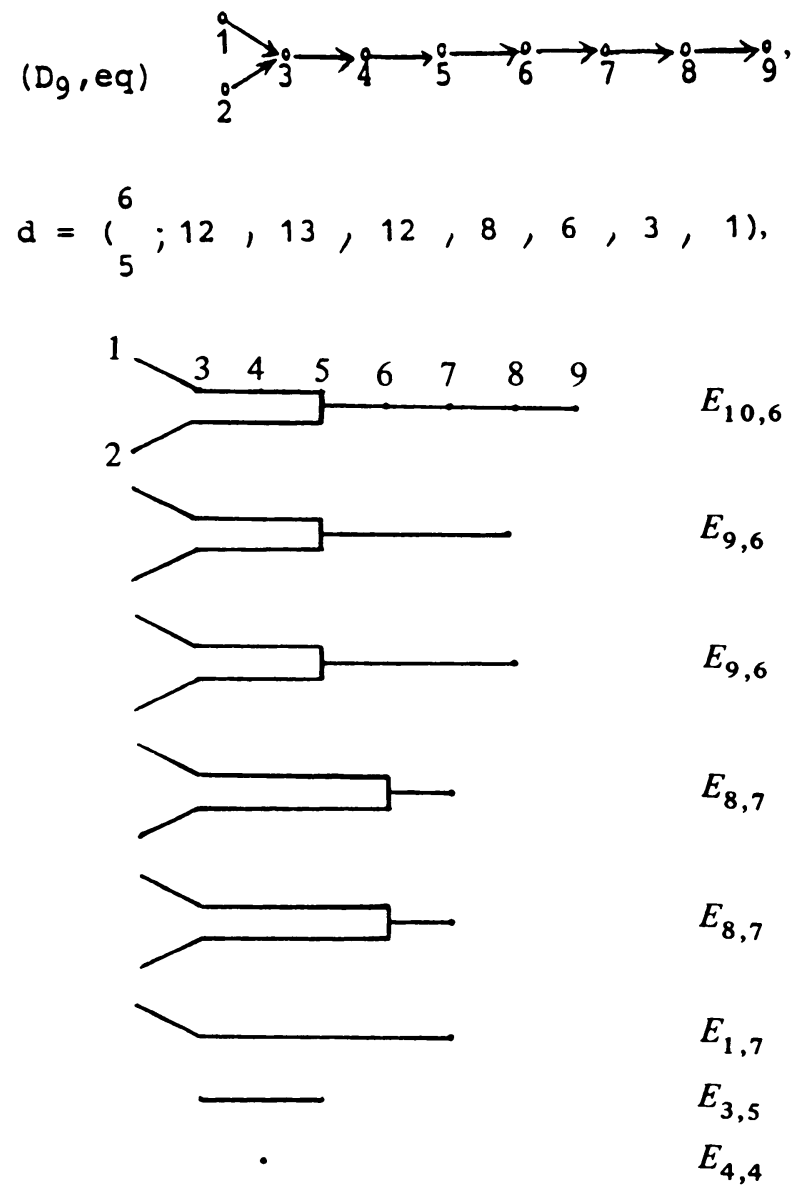




$$
\begin{gathered}
A=E_{10,6} \oplus 2 E_{9,6} \oplus 2 E_{8,7} \oplus E_{1,7} \oplus E_{3,5} \oplus E_{4,4}, \\
I_{d}^{\prime}=\{(8,6)\}, \quad I_{d}^{\prime \prime}=\{(3,5),(1,7)\}, \quad\left|I_{d}\right|=3, \\
\tau=\text { distinct ind. in } A=6, \quad n=9 .
\end{gathered}
$$

Construction $\psi$ for the codim. 1 orbits:

$$
\begin{gathered}
(8,6) \in I_{d}^{\prime}: \text { Case 1, Table I, } A=E_{9,6} \oplus E_{8,7} \oplus \bar{A}, \\
B_{8,6}=E_{8,6} \oplus E_{9,7} \oplus \overline{A ;} \\
(3,5) \in I_{d}^{\prime \prime}: \text { case 1, Table II, } A=E_{3,5} \oplus E_{4,4} \oplus \bar{A}, \\
B_{3,5}=E_{3,4} \oplus E_{4,5} \oplus \overline{A ;} \\
(1,7) \in I_{d}^{\prime \prime}: \text { case 8, Table II (special case), } \\
A=E_{8,7} \oplus E_{1,7} \oplus \bar{A}, \quad B_{1,7}=E_{1,7} \oplus E_{2,7} \oplus E_{1,6} \oplus \bar{A} .
\end{gathered}
$$

6. The ring of semi-invariant polynomials $\mathscr{R}_{d}\left(D_{n}, \mathrm{eq}\right)$. We first recall some known facts. Let $K$ be an algebraically closed field and $\rho$ a rational representation of a connected algebraic group $G$ on a finite-dimensional vector space over $K$. Then $G$ acts on the polynomial ring $K[V]$ of $V$ via the action

$$
f^{g}(v)=f\left(\rho\left(g^{-1}\right) v\right), \quad g \in G, v \in V .
$$

A polynomial $f \in K[V]$ is said to be semi-invariant (or relative invariant) if $f^{g}=\chi(g) f(\chi$ a character of $G)$. The ring of semi-invariants of the representation $(G, \rho, V)$ is by definition the ring generated by the semi-invariant polynomials. $(G, \rho, V)$ is called prehomogeneous if there is an open dense orbit $O_{\max } \subset V$. In this case it has been proved by M. Sato and T. Kimura (cf. [13]) that the reduced equations $f_{i}=0$ of the codim. 1 components in $V \backslash O_{\max }$ are such that the $f_{i}$ 's are an algebraically independent system of semi-invariant polynomials which generate the ring of semi-invariants.

Let us now consider the Dynkin diagram $D_{n}$ equioriented. For a given dimension $d(\operatorname{see}(*))$ we have the variety

$$
L_{d}=L_{d}\left(D_{n}, \text { eq }\right)=\operatorname{Hom}_{K}\left(V_{1}, V_{3}\right) \times \prod_{t=2}^{n-1} \operatorname{Hom}_{K}\left(V_{t}, V_{t+1}\right)
$$

( $V_{t}$ vector spaces over $K$ of dimension $d_{t}$ ) on which the group $G_{d}=\prod_{t-1}^{n} \mathrm{GL}\left(V_{t}\right)$ acts. We denote by $\mathscr{R}_{d}\left(D_{n}\right.$, eq $)$ the corresponding ring of semi-invariants. The representation of $G_{d}$ on $L_{d}$ is prehomogeneous and the codim. 1 components in $L_{d} \backslash O_{\max }$ are exactly the closure of the codim. 1 orbits in $L_{d}$ (which have been classified in \$5). In view of the Sato-Kimura theorem we want to compute, for every codim. 1 orbit, the equation of its closure. If we fix bases in the $V_{t}$ 's, a representation $X \in L_{d}$ is given by a $(n-1)$-tuple of matrices $\left(X_{1}, X_{2}, \ldots, X_{n-1}\right), X_{1}$ a $d_{3} \times d_{1}$ matrix, $X_{t}$ a $d_{t+1} \times d_{t}$ matrix, $2 \leqslant t \leqslant n-1$. Then the map $\varphi_{i j}^{X}, i \neq j$, defined in $\S 1$ is expressed by a matrix $Y_{i j}$ which, with a suitable choice of the bases for the spaces involved, can be written in terms of the $X_{t}$ 's and their compositions. For any 
$h<k \leqslant n$ let us define the $d_{k} \times d_{h}$ matrix

$$
\begin{aligned}
& X_{h k}=X_{k-1} \cdot X_{k-2} \cdots X_{h}, \quad h \neq 1, h<k, \\
& X_{1 k}=X_{k-1} \cdot X_{k-2} \cdots X_{3} \cdot X_{1}, \quad h=1 .
\end{aligned}
$$

Then we have

$$
\begin{aligned}
\varphi_{i j}^{x}: V_{i} & \rightarrow V_{j}, \quad Y_{i j}=X_{i j}, \quad i<j, \\
\varphi_{n+1, j}^{x}: V_{1} \oplus V_{2} & \rightarrow V_{j}, \quad Y_{i j}=\left[X_{1 j} X_{2 j}\right], \quad i=n+1
\end{aligned}
$$

(and the choice of the bases is obvious). If $i>j$ and $i \neq n+1$ we have the map

$$
\varphi_{i j}^{x}: V_{1} \oplus V_{2} \oplus V_{j} \rightarrow\left(V_{j} \oplus V_{j}^{\prime} \oplus V_{j}^{\prime \prime}\right) / \Delta \oplus V_{i}
$$

where $V_{j}^{\prime}$ and $V_{j}^{\prime \prime}$ are copies of $V_{j}$. Let $w_{1}, \ldots, w_{d}$, be the given basis in $V_{j}$ and $w_{h}^{\prime}, w_{h}^{\prime \prime}$, $h=1, \ldots, d_{j}$ the same base in the copies $V_{j}^{\prime}, V_{j}^{\prime \prime}$. Then a basis in $\left(V_{j} \oplus V_{j}^{\prime} \oplus V_{j}^{\prime \prime}\right) / \Delta$ is given by $\bar{w}_{1}, \ldots, \bar{w}_{d}, \bar{w}_{1}^{\prime}, \ldots, \bar{w}_{d}^{\prime}$ where $\bar{w}_{h}$ (resp. $\bar{w}_{h}^{\prime}$ ) is the class of $w_{h}$ (resp. $w_{h}^{\prime}$ ) in the quotient space. Then the matrix corresponding to $\varphi_{i j}^{x}$ is

$$
Y_{i j}=\left[\begin{array}{ccc}
X_{1 j} & 0 & -I \\
0 & X_{2 j} & -I \\
0 & 0 & X_{j i}
\end{array}\right], \quad i>j
$$

( $I$ the identity matrix of size $d_{j}$ ).

We are interested in the matrices $Y_{i j}$ only if $(i, j) \in I_{d}$. In this case $Y_{i j}$ is a square matrix and we define

$$
D_{i j}=\operatorname{det} Y_{i j}, \quad(i, j) \in I_{d} .
$$

THEOREM 6.5. If $K$ is an infinite field then:

(1) The $D_{i j}$ 's, $(i, j) \in I_{d}$, are a set of algebraically independent semi-invariant polynomials.

(2) Any semi-invariant polynomial is a product of the $D_{i j}$ 's and $\mathscr{R}_{d}\left(D_{n}\right.$, eq $)=K\left[D_{i i}\right]$.

Proof. Let $(i, j) \in I_{d}$ and consider the corresponding polynomial $D_{i j}$, which is not identically zero on $L_{d}$ since it does not vanish on the open orbit $O_{\max }$ (in fact $\varphi_{i j}^{A}$ is an isomorphism for $A \in O_{\max }$, cf. Corollaries 4.4, and 4.5). $D_{i j}=0$ on $\bar{O}_{i j}$ $=\overline{\psi(i, j)}$ as a consequence of Theorem 5.1(a) and $D_{i j}$ does not vanish on any other codim. 1 orbit of $L_{d}$ as a consequence of Theorem 5.1(b). At this point we first prove the theorem if $K$ is algebraically closed, showing that $D_{i j}=0$ is the reduced equation of $\bar{O}_{i j}$, and then we extend the result to the case of an infinite field $K$, passing through its algebraic closure $\bar{K}$. The argument is omitted since it is completely similar to the one given in [2, Theorem 0.1].

\section{REFERENCES}

1. S. Abeasis, On the ring of semi-invariants for the representations of an equioriented quiver of type $A_{n}$, Boll. Un. Mat. Ital. (6) 1-A (1982), 233-240.

2. Codimension 1 orbits and semi-invariants for the representations of an oriented graph of type $\mathscr{A}_{n}$, Trans. Amer. Math. Soc. 282 (1984), 463-485.

3. S. Abeasis and A. Del Fra, Degenerations for the representations of an equioriented quiver of type $A_{m}$, Boll. Un. Mat. Ital. Suppl. Algebra e Geometria 2 (1980), 158-171.

4. , Degenerations for the representations of a quiver of type $A_{m}, \mathrm{~J}$. Algebra (to appear). 
5. S. Abeasis, A. Del Fra and H. Kraft, The geometry of representations of $A_{m}$, Math. Ann. 256 (1981), 401-418.

6. I. N. Bernstein, I. M. Gelfand and V. A. Ponomarev, Coxeter functors and Gabriel's theorem, Uspekhi Mat. Nauk 28 (1973), 19-33; English transl., Russian Math. Surveys 28 (1973), 17-32.

7. V. Dlab and G. M. Ringel, Indecomposable representations of graphs and algebras, Mem. Amer. Math. Soc. No. 173 (1976).

8. P. Gabriel, Représentations indécompasables, Sém. Bourbaki, 26e année (1973/1974), Exp. 444, Lecture Notes in Math., Vol. 431, Springer, Berlin, 1975, pp. 143-169.

9. D. Happel, Relative invariants and subgeneric orbits of quivers of finite and tame type, Lecture Notes in Math., vol. 903, Springer, Berlin and New York, 1981, pp. 116-124.

10. Relative invariants and subgeneric orbits of quivers of finite and tame type, J. Algebra 78 (1982), 445-459.

11. V. Kac, Infinite root systems, representations of graphs and invariant theory, Invent. Math. 56 (1980), 57-92.

12. __ Infinite root systems, representations of graphs and invariant theory. II, J. Algebra 77 (1982), $141-162$.

13. M. Sato and T. Kimura, A classification of irreducible pre-homogeneous vector spaces and their relative invariants, Nagoya Math. J. 65 (1977), 1-555.

Dipartimento di MATEMatica, II Universitá di Roma, Roma, Italy 